\title{
Empirical Evaluation of Defect Projection Models for Widely-deployed Production Software Systems
}

\author{
Paul Luo Li, Mary Shaw, Jim Herbsleb, \\ Bonnie Ray*, and P. Santhanam* \\ August 2004 \\ CMU-ISRI-04-130
}

\author{
Institute for Software Research International \\ School of Computer Science \\ Carnegie Mellon University \\ Pittsburgh PA, 15213
}

This paper is an expanded version of the paper titled: Empirical Evaluation of Defect Projection Methods for Widely-deployed Production Software Systems, in SIGSOFT 2004/FSE-12, November, 2004.

*Bonnie Ray and P. Santhanam are researchers at the Center for Software Engineering at IBM T. J. Watson Research Center.

This research was supported by the National Science Foundation under Grand CCR-0086003, by the Sloan Software Industry Center at Carnegie Mellon University, and by the NASA High Dependability Computing Program under cooperative agreement NCC-2-1298. 
Keywords: Empirical Studies, Metrics, Reliability Engineering, Defect modeling, empirical research, COTS, open source software, maintenance resource planning, software insurance 


\begin{abstract}
Defect-occurrence projection is necessary for the development of methods to mitigate the risks of software defect occurrences. In this paper, we examine user-reported software defect-occurrence patterns across twenty-two releases of four widely-deployed, business-critical, production, software systems: a commercial operating system, a commercial middleware system, an open source operating system (OpenBSD), and an open source middleware system (Tomcat). We evaluate the suitability of common defect-occurrence models by first assessing the match between characteristics of widely-deployed production software systems and model structures. We then evaluate how well the models fit real world data. We find that the Weibull model is flexible enough to capture defectoccurrence behavior across a wide range of systems. It provides the best model fit in 16 out of the 22 releases. We then evaluate the ability of the moving averages and the exponential smoothing methods to extrapolate Weibull model parameters using fitted model parameters from historical releases. Our results show that in 50\% of our forecasting experiments, these two naïve parameter-extrapolation methods produce projections that are worse than the projection from using the same model parameters as the most recent release. These findings establish the need for further research on parameter-extrapolation methods that take into account variations in characteristics of widely-deployed, production, software systems across multiple releases.
\end{abstract}




\section{INTRODUCTION}

Defect occurrences not only create problems for software consumers, but also cause problems in maintenance planning for software producers. The costly consequences of defect occurrences have increased interest in insuring software consumers against the associated risks.

Defect-occurrence projection is crucial to the development of methods for managing the risks associated with defect occurrences. Accurate defect-occurrence projections can help software maintenance planners to better allocate resources and will be a major step towards novel risk-mitigation techniques for software consumers, such as software insurance.

We examine software systems that businesses are increasingly dependent upon. These systems are multirelease, multi-platform, and widely-deployed, such as COTS and open source software. It is generally accepted that these widely-deployed, production, software systems (WPSSs) are not defect free and that there is a need to manage the risks associated with the defect occurrences.

We empirically address two questions that are important for defect-occurrence projection:

- Is there a type of defect occurrence model that provides a good fit to defect-occurrence patterns across multiple releases and in many organizations?

- Given such a model, how can model parameters for a new release be extrapolated using historical information?

Our findings provide a basis for a defect-occurrence projection method for WPSSs that is robust across many organizations and development styles.

We use data from a diverse sample of WPSSs including two different types of software systems (middleware and operating systems) developed with two different development styles (commercial and open source). We gather data from twenty-two releases: eight releases of a commercial operating system, three releases of a commercial middleware system, eight releases of an open source operating system (OpenBSD), and three releases of an open source middleware system (Tomcat).

We examine characteristics of WPSSs that can change between releases and that may cause variations in defect-occurrence patterns. The characteristics we consider are release content, development process, adoption and usage patterns, and software and hardware configurations in use. These considerations are not modeled well in prior research in this field.

We examine how parameterizations of a set of candidate defect-occurrence models taken from the literature account for possible variations in defect-occurrence patterns across multiple releases and how two commonlyused naïve parameter-extrapolation methods account for the variations. We hypothesize:

- The Weibull model is better than other candidate models at modeling defect-occurrence patterns for multiple releases of WPSSs.

- Naïve parameter-extrapolation methods, moving averages and exponential smoothing, extrapolate model parameters that produce inadequate defect-occurrence projections for new releases of WPSSs.

Determining the preferred defect model is important for defect-occurrence projection because it may allow us to understand and quantify the effects of changes in characteristics of WPSSs across multiple organizations and development styles. Verifying that naïve parameter-extrapolation methods are inadequate is important because it will motivate additional research questions on new methods for parameter extrapolation. Our results show that the Weibull model is the preferred model and that naïve parameter-extrapolation methods are inadequate.

We begin by providing background and descriptions in Section 2. We present analyses that support our two hypotheses and the empirical results in Sections 3 and 4. We conclude with validity issues and future work in Section 5.

\section{BACKGROUND AND DESCRIPTIONS}

We are interested in real-world software systems deployed today that are of key business interest to users, such that there are users who pay for maintenance contracts and who may be willing to pay to insure against defect occurrences. 


\subsection{Defect occurrence}

We define a defect occurrence as a user-reported problem that requires developer intervention to correct. This is the observable event of interest for both maintenance and insurance purposes.

The operational definition of a defect occurrence varies across organizations. In this paper, we use the same approach to analyze defect occurrences in different organizations and show that our approach is resilient to organizational differences. The commercial software development organizations measure faults and failures, described in Section 2.2.1, while open source software projects track user-submitted bug-reports, described in Section 2.2.2. Our findings support the idea that a common defect-occurrence projection method for WPSSs can be used across many organizations and development styles.

We are interested the defect-occurrence pattern, which is the rate of defect occurrence as a function of time over the lifetime of a release. We define the lifetime of a release as the duration of time between when a release becomes generally available and when there are no defect occurrences reported to the software development organization for three consecutive time intervals. Determining the lifetime of a release is discussed in detail in [Appendix C].

Our focus on the defect occurrence pattern is different from previous research on the total number of defects [5] and the normalized defect-occurrence rate. The normalized defect-occurrence rate is the rate of defect occurrences normalized with respect to the number of deployed systems and the usage amount over the lifetime of a release [4][18][19][20][28]. Knowing only the total number of defects is inadequate because resource allocation for maintenance planning and cash reserve management for insurance both require knowledge of how many defects are going to occur in a given time period. The normalized defect-occurrence rate is unsuitable because it requires accurate measurements of deployment and usage patterns. As we explain in the next section, unknown deployment and usage patterns are properties of WPSSs.

\subsection{Widely-deployed production software systems}

We are interested in widely-deployed, production, software systems (WPSSs), which are software systems with the following properties:

- The software system is used in many software and hardware configurations (some unforeseen).

- The deployment and usage patterns of the software system are unknown.

- The development process of software system has constraints (such as scheduling and resource constraints).

- The contents of the software system change over time.

- The software system has multiple releases.

\subsubsection{Commercial software systems}

Nearly all COTS software systems have the properties of WPSSs. COTS software systems are not developed with one client in mind but rather to be sold on the open market and to be used by many clients [2]. The systems are typically built to run on multiple hardware platforms, to be compatible with many different hardware devices, and to be compatible with many other software systems. Constraints exist on the development process, such as pre-set release dates and limited resources. The software development organization has limited information on who is going to purchase the software system and puts out successive versions of the software system, which implements new functionality and improvements [12].

The two COTS software systems we examine are developed by two different divisions of IBM. The operating system is a mature product with many years of presence in the marketplace. The commercial middleware system has a few years of deployment history and a growing customer base.

The defect-occurrence data collected are code-related problems discovered and reported by customers after deployment. A more detailed description of the data collection process is in [Appendix E]. The defect-occurrence data for the operating system contain unique field defects that led to code changes by the product support organization. The defect-occurrence data for the middleware system contain all field defects (which may not be unique) that lead to code changes. The defect-occurrence data are processed and aggregated, so for each data set we use the interval in that data set. The time interval for the middleware system is a month and the time interval for the operating system is a quarter. 


\subsubsection{Open source software systems}

Open source software systems have all the properties of WPSSs. A consortium of user-developers with different needs (generally with one person or a core group leading development) develops an open source software system. A successful project has diverse user-developers that develop and test the software system, port the software system to many platforms, make it operable with many devices, and make it compatible with other software systems. Hence, the number of active user-developers constrains an open source project. The software system can be downloaded and used anonymously, so there is limited knowledge about the users. Open source software systems usually evolve in successive releases to satisfy the needs of its user-developers [25].

The open source software systems we examine are developed by successful open source projects with many user-developers from around the world. The open source operating system is OpenBSD. OpenBSD is a Unix-like operating system that emphasizes portability, standardization, correctness, proactive security, and integrated cryptography [21]. The open source middleware system is Tomcat, which is one of the products developed by the Jakarta Project. Tomcat is the servlet container used in the official reference implementation for the Java Servlet and JavaServer Pages technologies [7].

The defect-occurrence data collected are bug reports submitted by users via the web based bug-tracking system. A more detailed description of the data collection process is in [Appendix E]. Unlike users of a commercial software system, user-developers of an open source software system can obtain the software system anytime during development and submit bug reports. Many bugs are duplicates, user mistakes, or otherwise invalid submissions that do not require code changes. However, we include all user-reported bug reports in our data set because a member of the core development team examines each bug submission and decides upon a course of action. The time interval used for both open source software systems is a month.

\subsubsection{Related work}

Much of the prior research in software reliability has been conducted on systems where the testing environment and the deployment environment are similar. Similarities like software and hardware configurations and usage patterns have allowed researchers to extend defect-occurrence patterns from development into the field. Lyu in [16] provides a comprehensive review of previous works. Lyu explains model origins, states modeling assumptions, and classifies commonly used reliability models. We borrow mathematical models and statistical tools from prior research, however extending defect-occurrence patterns is inappropriate for WPSSs given properties discussed in Section 2.2.

Current efforts to certify software are very similar to prior research in software reliability engineering. Voas advocates certifying commercial software for use in a customer's environment [28], and Wallnau et. al. at the Software Engineering Institute are conducting research on predictable assembly from certified components [14]. Both approaches test software in the customer's environment then extend results into usage. These approaches account for several environmental variables and make statistical guarantees about various properties. However, we feel that cost, variance, and unforeseen confounds at the single-customer level might make such methods impractical. We do however, leverage relationships between characteristics of software systems and defect occurrences discovered in their research.

Several on-going research efforts examine defect occurrences across multiple releases. Ostrand and Weyuker use software content and development process measures to predict faulty files in multiple releases of two software systems at AT\&T [22]. Their model predicts the top 20\% faultiest files, which they show to capture around $80 \%$ of faults found during development and in the field. The COQUALMO project at USC uses COCOMO II data to estimate the total number of defects in a software system [5]. Their model uses size metrics and various process modifiers. The process modifiers measure aspects of the defect injection process and the defect removal process. Jones et. al at Nortel use the percentage of deployed systems with a module installed as a surrogate for usage along with software content measures to predict the likelihood that a module will be faulty in the field [10]. They correctly identify approximately $70 \%$ of the faulty modules while misidentifying less than $26 \%$ of the faulty modules. These research projects contribute knowledge about the important predictors of defect occurrences. However, none of the projects examines the defect-occurrence pattern. As noted in Section 
2.1, risk mitigation techniques need to know both the expected total number of defect occurrences as well as when those defect occurrences happen in the lifetime of a release.

Mockus et. al. use software content and development process information captured in the change management and CVS systems to predict the amount of repair effort and the delay until the effort is needed for eleven releases of a telecommunications software system at Avaya [17]. They assume that field repair effort is proportional to development effort and estimate a delay factor and an effort multiplier. Our work is similar. However, our focus is on defect-occurrence patterns, while the focus at Avaya is on effort.

Popstajanova et. al in [23] uses architecture, utilization, and control flow information to predict the likelihood of defect occurrences. Unlike their white box approach, our approach is black box.

Previous works have also compared defect-occurrence models. Jones compares various models and the MLE and least squares model fitting methods for ten releases of a telecommunications software system at Northern Telecom and Bell Canada [9]. He concludes that the Logarithmic model, fitted using the least squares method, produces the best results and that the least squares method is superior to the MLE method overall. Jones considers a Weibull process model. Wood compares eight mathematical models fitted using the least squares method for four releases of a software system at Tandem computers [28]. Wood considers the Weibull model but finds that the Exponential is the best model in his environment. Their works lack an underlying theory explaining why a model is superior in their environment, and they do not replicate their experiment at other organizations. We develop a theory for the causes of variation in defect-occurrence patterns for WPSSs and compare models across multiple releases, multiple organizations, and multiple products.

There appears to be no published work on projecting defect-occurrence patterns for open source projects. We note that software systems are becoming so complex and expensive that few organizations have the resources to build custom systems. More and more organizations reply on COTS or open source software systems, which is the focus of this paper.

\subsection{Characteristics of WPSSs}

Content, development process, deployment and usage patterns, and software and hardware configurations in use are characteristics of WPSSs that tend to change, sometimes dramatically, between releases. The effect of changes on defect-occurrence patterns will dictate which defect-occurrence model is best suited to model defectoccurrence patterns in multiple releases and which parameter-extrapolation methods are effective. We use results from previous work in software reliability engineering and intuitive arguments to provide evidence that the characteristics listed above can influence defect-occurrence patterns across multiple releases.

\subsubsection{Content}

Software content affects defect-occurrence patterns across multiple releases [11][17]. Successive releases incrementally add features and implement internal changes, such as refactoring. Some modifications may be more difficult and defect prone than others, which may cause more defect occurrences over a longer time period. Depending on the similarity of content changes, the defect-occurrence pattern of a release may be similar to one release but be substantially different from another.

\subsubsection{Development process}

The development process affects defect-occurrence patterns across multiple releases [12][7]. Although the development process of most organizations changes slowly over time, when combined with other factors the development process may have significant impact on defect-occurrence patterns. Insufficient and varying testing resources and schedule pressure may lead to ineffective defect removal. This can cause defects to linger in the system and can cause blocking, which occurs when one defect masks the presence of other defects. However, problems with the development process may not be present to the same degree in every release, which may cause defect-occurrence patterns to vary.

\subsubsection{Deployment and usage patterns}

Deployment and usage patterns affect defect-occurrence patterns across multiple releases [3][16]. The total number of deployed systems and the pattern of deployment dictate how much usage and how many usage patterns are possible. Deployment may be different for each release because users cannot be forced to adopt the latest 
release. Some users may adopt every release, while others may only adopt releases that contain important functionality. In addition, some users may adopt a release immediately, while other may delay adoption. The usage patterns may also be different from release to release and from user to user. Some users may not heavily exercise the software until the software has shown to be satisfactory under normal operating conditions. Deployment and usage patterns dictate how heavily the system is exercised, which may cause variations in defect-occurrence patterns.

\subsubsection{Software and hardware configurations in use}

Software and hardware configurations in use affect defect-occurrence patterns across multiple releases [14][28]. The software development organization has limited knowledge about users, and thus may not be able to test all possible hardware and software configurations. Furthermore, comprehensive testing may not be feasible given economic and scheduling constraints. Therefore, the software may have defects that are specific to certain configurations, software interactions, and other special conditions, such as malicious attacks.

\section{DEFECT-OCCURRENCE MODELS}

A single type of defect-occurrence model may be able to model defect-occurrence patterns across multiple releases of a wide variety of WPSSs. We believe such a model exists despite possible changes in characteristics discussed in Section 2.3 because of similarities between releases and properties common to all WPSSs. Bassin and Santhanam at IBM have shown that successive releases have similarities in the functionalities implemented, the development organization, and users with similar usage patterns [1]. All WPSSs also share common properties mentioned in Section 2.2.

In order for a model to be widely applicable, it needs to have parameterization that can account for variations in defect-occurrence patterns. In this section, we develop and use real-world data to empirically test the hypothesis that the Weibull model is better than other candidate models in modeling defect-occurrence patterns for multiple releases of WPSSs.

\subsection{Candidate models}

We are interested in the defect-occurrence pattern. Therefore, we are interested in models that model the number of defect occurrences in each time period over the lifetime of a release. We consider the Exponential model, the Gamma model, the Logarithmic model, the Power model, and the Weibull model. These models are promising because prior research in software reliability engineering has shown each model to be effective at modeling defect-occurrence patterns at a software development organization [9][16][28]. Each model is parametric. The number of defect occurrences during the t-th time interval is determined by the model parameterization and the current time interval. The number of defect occurrences within a time interval is modeled as a non-homogenous Poisson process with a stationary defect rate $\lambda(\mathrm{t})$. Table 1 lists the models. Lyu [16] provides details about the models, including researchers who have developed and applied the models in practice.

Table 1. Candidate models

\begin{tabular}{|c|c|c|c|}
\hline Model type & Model name & Model form & Researchers/users of the model \\
\hline Exponential & Non-homogenous Poisson process model & $\lambda(\mathrm{t})={\mathrm{N} \alpha \mathrm{e}^{-\alpha \mathrm{t}}}^{\text {Goel \& Okumoto }}$ \\
\hline Weibull & Weibull & $\lambda(\mathrm{t})=\mathrm{N \alpha} \alpha \mathrm{t}^{\alpha-1} \mathrm{e}^{-\beta \mathrm{t}^{\alpha}}$ & Schick-Wolverton \\
\hline Gamma & S-shaped reliability growth model & $\lambda(\mathrm{t})=\mathrm{N} \beta^{\alpha} \mathrm{t}^{\alpha-1} \mathrm{e}^{-\beta \mathrm{t}}$ & Yamada, Ohba \& Osaki \\
\hline Power & Duane Model & $\lambda(\mathrm{t})=\alpha \beta \mathrm{e}^{-\beta \mathrm{t}}$ & Duane \\
\hline Logarithmic & Musa-Okumoto logarithmic Poisson model & $\lambda(\mathrm{t})=\alpha(\alpha \beta \mathrm{t}+1)^{-1}$ & Musa-Okumoto \\
\hline
\end{tabular}

\subsection{The Weibull model}

The Weibull model can account for different increasing and decreasing trends, which reflect initial increases and eventual decreases in defect occurrences. We generally expect early defect occurrences to show a primarily increasing trend as users migrate to the release, exercise the software, and report defects, and we expect later 
defect occurrences to show a primarily decreasing trend as the rate of adoption declines and the release becomes more reliable due to defect removal.

The Weibull model has three parameters $\mathrm{N}, \alpha$, and $\beta$. Intuitively, the Weibull model can be broken down into three interacting pieces: $\mathrm{N}$, representing the total number of defect occurrences in the lifetime of the release, a generally increasing component $\alpha \beta \mathrm{t}^{\alpha-1}$, which dominates early, and a decreasing component $\mathrm{e}^{-\beta \mathrm{t}^{\alpha}}$, which dominates as time increases.

$\mathrm{N}$ can be different for each release, which can account for differences in the total number of defect occurrences between releases. The differences may be caused by changes in software content or development processes.

In general, $\alpha \beta \mathrm{t}^{\alpha-1}$ increases as a function of time and can account for increases in defect occurrences. The rate of growth is controlled by a combination of the $\alpha$ and $\beta$ parameters. The increasing component is flexible enough to describe both concave and convex increasing patterns. Concave increasing patterns can occur when the growth in the rate of defect occurrences is faster at the beginning of the release, which may occur if many users quickly adopt and use a release. Convex increasing patterns can occur when the rate of defect occurrences increase slowly. This may occur if users slowly migrate to the release or if constraints on development and problematic content cause blocking, which prevents defects from being discovered.

The term $\mathrm{e}^{-\beta \mathrm{t} \alpha}$ decreases as a function of time and can account for decreases in defect occurrences. Again, the rate of decrease is controlled by a combination of the $\alpha$ and $\beta$ parameters. The decreasing component can describe concave or convex decreasing patterns. Convex decreasing patterns can occur when the rate of defect occurrences decrease rapidly, which may occur if there is fast migration to a new release. Concave patterns can occur when the rate of defect occurrences decrease slowly, which may occur if defect occurrences remain high over a longer time period due to constraints on development or problematic content.

It is reasonable to expect a model with parameterization that can describe varying defect-occurrence patterns such as the Weibull model to be better than other candidate models. The Exponential, the Power, and the Logarithmic models do not have both decreasing and increasing components. They cannot describe the interplay of increasing and decreasing trends. Although the Gamma model has both increasing and decreasing components, its decreasing component is generally convex. Thus, the Gamma model is unable to describe situations in which the decreasing pattern is concave. Kenny [11] has studied the interaction of increasing and decreasing trends in defect-occurrence patterns in commercial software systems and recommends using the Weibull model to model defect-occurrence patterns.

We hypothesize that the Weibull model is better than other candidate models at modeling defect-occurrence patterns for multiple releases of WPSSs.

\subsection{Model fitting and model selection}

We fit the best set of parameters for each candidate model for each release using Non-linear Least Squares (NLS) regression then compare the candidate models using the Akaike Information Criterion (AIC) model selection criterion[27].

NLS is a well-established model fitting procedure that selects model parameters by minimizing the square of the difference between fitted values and actual values [27]. It is widely used in defect modeling research [9][28]. We use the open source statistical computing package R [24]. After we select the best parameters for each candidate model for a given release, we use the AIC model selection criterion to evaluate the fit of the different candidate models; lower AIC scores are better. The AIC score is defined as:

$$
\mathrm{AIC}=\mathrm{n} \log \sigma^{2}+2|\mathrm{~S}|
$$

where $\sigma^{2}$ is the residual squared error divided by the difference of the number of observations, $n$, and the number of model parameters, S [27]. The AIC model selection criterion penalizes models with more parameters to offset the advantage models with more parameters have in comparisons.

Our hypothesis stated in Section 3.2 would be supported if the Weibull model consistently produces lower AIC score. 
Tables 2-5 present the AIC scores. The best AIC scores for each release are highlighted (shaded cells highlight the best AIC scores among fitted candidate models). INF and Singular Gradient indicate that the modelfitting algorithm is unable to fit parameters for the model, which suggests that the model is inappropriate. A detailed explanation of the failure to fit model parameters and why it suggests a model is inappropriate is given in [Appendix B].

Table 2. AIC scores for commercial OS

\begin{tabular}{|c|c|c|c|c|c|}
\hline Release/ Model & Exponential Model & Weibull Model & Gamma Model & Power Model & Logarithmic Model \\
\hline$i$ & 158 & $\mathbf{1 3 1}$ & 144 & 164 & 160 \\
\hline$i+1$ & 119 & $\mathbf{1 1 0}$ & 111 & 131 & 126 \\
\hline$i+2$ & 159 & $\mathbf{1 5 0}$ & 155 & 113 & 169 \\
\hline$i+3$ & $\mathbf{1 0 4}$ & 105 & 111 & 121 & 109 \\
\hline$i+4$ & 116 & 111 & $\mathbf{1 0 9}$ & 105 & 104 \\
\hline$i+5$ & 104 & $\mathbf{8 7}$ & 89 & $\mathbf{6 2}$ & 6 \\
\hline$i+6$ & $\mathbf{6 2}$ & 64 & 64 & $\mathbf{6 2}$ & Singular Gradient \\
\hline$i+7$ & Singular Gradient & $\mathbf{6 3}$ & $\mathbf{6 3}$ & 66 & \\
\hline
\end{tabular}

Table 3. AIC scores for commercial middleware system

\begin{tabular}{|c|c|c|c|c|c|}
\hline Release/ Model & Exponential Model & Weibull Model & Gamma Model & Power Model & Logarithmic Model \\
\hline$i$ & 153 & $\mathbf{1 3 4}$ & $\mathbf{1 3 4}$ & 163 & 157 \\
\hline$i+1$ & Singular Gradient & 173 & $\mathbf{1 7 1}$ & 195 & Singular Gradient \\
\hline$i+2$ & Singular Gradient & $\mathbf{1 1 6}$ & $\mathbf{1 1 6}$ & 129 & Singular Gradient \\
\hline
\end{tabular}

Table 4. AIC scores for open source OS

\begin{tabular}{|c|c|c|c|c|c|}
\hline Release Model & Exponential Model & Weibull Model & Gamma Model & Power Model & Logarithmic Model \\
\hline 2.7 & 162 & 91 & 103 & 176 & 171 \\
\hline 2.9 & 135 & 97 & 88 & 143 & 139 \\
\hline 3.0 & 110 & 83 & 90 & 113 & 112 \\
\hline 3.3 & 91 & 73 & 76 & 93 & 91 \\
\hline
\end{tabular}

Table 5. AIC scores for open source middleware system

\begin{tabular}{|c|c|c|c|c|c|}
\hline Release /Model & Exponential Model & Weibull Model & Gamma Model & Power Model & Logarithmic Model \\
\hline 3.3 & INF & $\mathbf{1 5 7}$ & $\mathbf{1 5 7}$ & 193 & 192 \\
\hline 4.0 & INF & $\mathbf{2 1 5}$ & 219 & 275 & 92 \\
\hline 4.1 & INF & $\mathbf{7 7}$ & 78 & 92 \\
\hline
\end{tabular}

Figure 1 shows fitted candidate models for a representative sample of releases from each of the four WPSSs. Due to confidentiality agreements, only the figures for open source software systems have numbered axes. Plots of all fitted candidate models are in [Appendix A].

Tables 2-5 show that the Weibull model has the best AIC score or one of the best AIC scores in 16 out of 22 or $73 \%$ of the releases. This is considerably better than the next best model, which is the Gamma model with the best AIC score or one of the best AIC scores in 8 out of 22 or $36 \%$ of the releases. Furthermore, since the AIC is a measure of deviance, it roughly follows a $\chi^{2}$ (chi-squared) distribution, which makes 4 a rough $95 \%$ confidence band around an AIC score. We note that the Weibull is within the 95\% confidence band of the best AIC score in all but one of the releases. These results support our hypothesis that the Weibull model is the preferred model. 


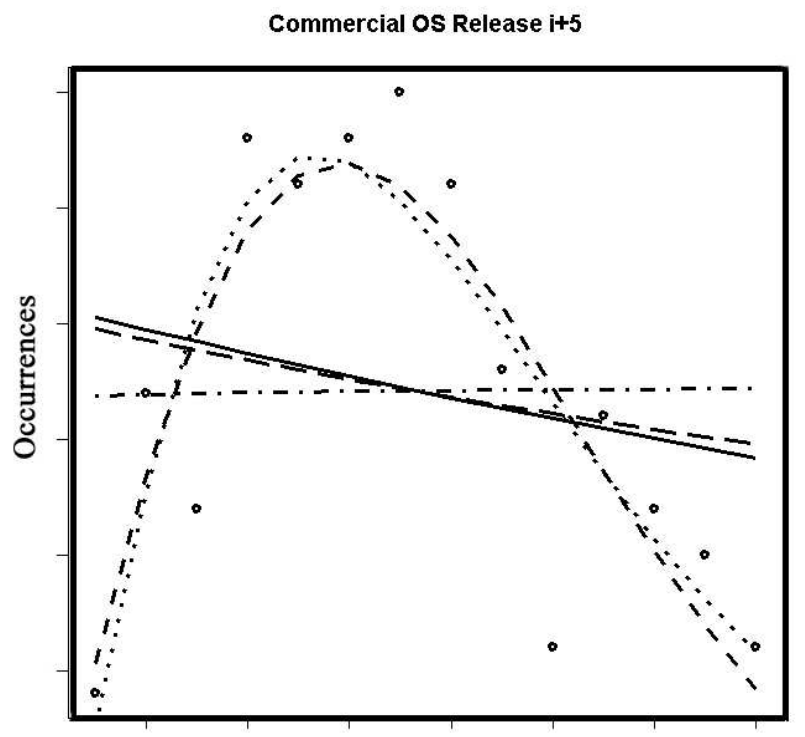

Time

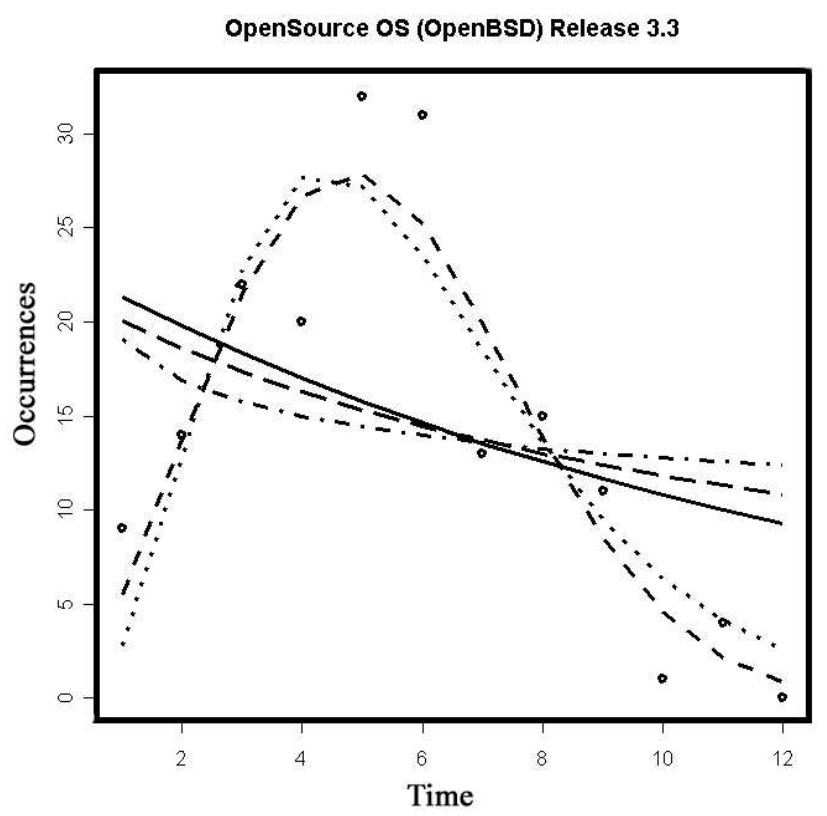

Commercial Middleware Release i+2

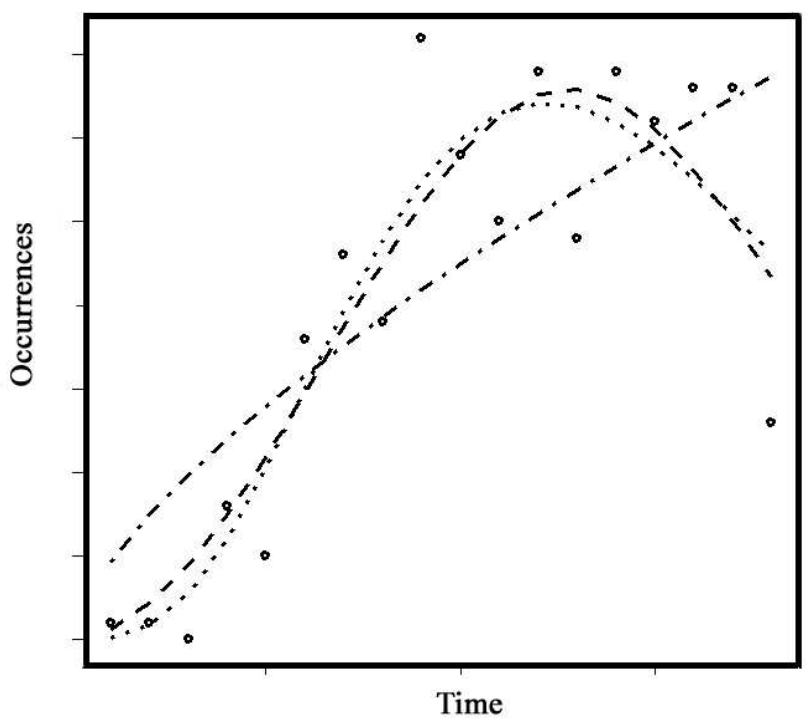

Open source MW system (Tomcat) Release 4.1

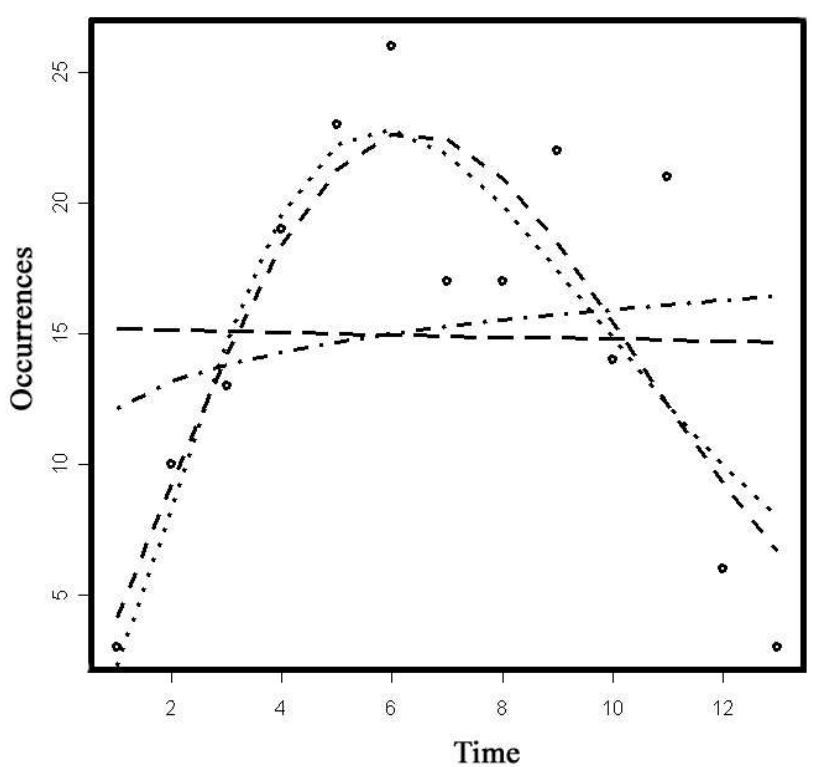

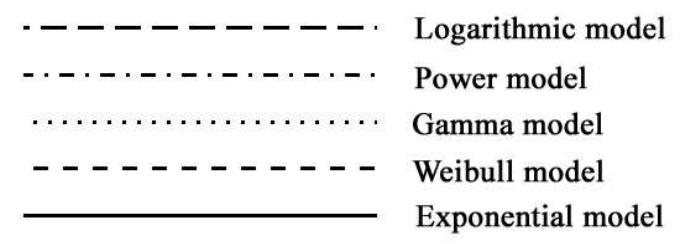

Figure 1. Samples of fitted candidate models

\subsection{Validation of the Weibull model}

We have shown that the Weibull is better than other candidate models. However, we still need to show that the Weibull model adequately describes the defect-occurrence pattern. We use the Theil forecasting statistic to validate the Weibull model. 
The Theil statistic compares the forecast for each time interval $i$ against a no-change forecast based on the previous time interval's value [26].

$$
U^{2}=\frac{\Sigma\left(P_{i}-A_{i}\right)^{2}}{\Sigma A_{i}^{2}}
$$

The Theil statistic $U$ is greater or equal to zero. The term $P_{\mathrm{i}}$ is the projected change and $A_{\mathrm{i}}$ is the actual change in interval $i$. A Theil statistic of zero indicates perfect forecasts with $P_{i}=A_{i}$. A Theil statistic of one indicates that forecasts are no better than no-change forecasts with $P_{i}=0$. Values greater than one indicate forecasts are worse than no-change forecasts.

The Theil statistics shown in Figure 2 indicate that the best-fit Weibull is always better than the no-change forecast.

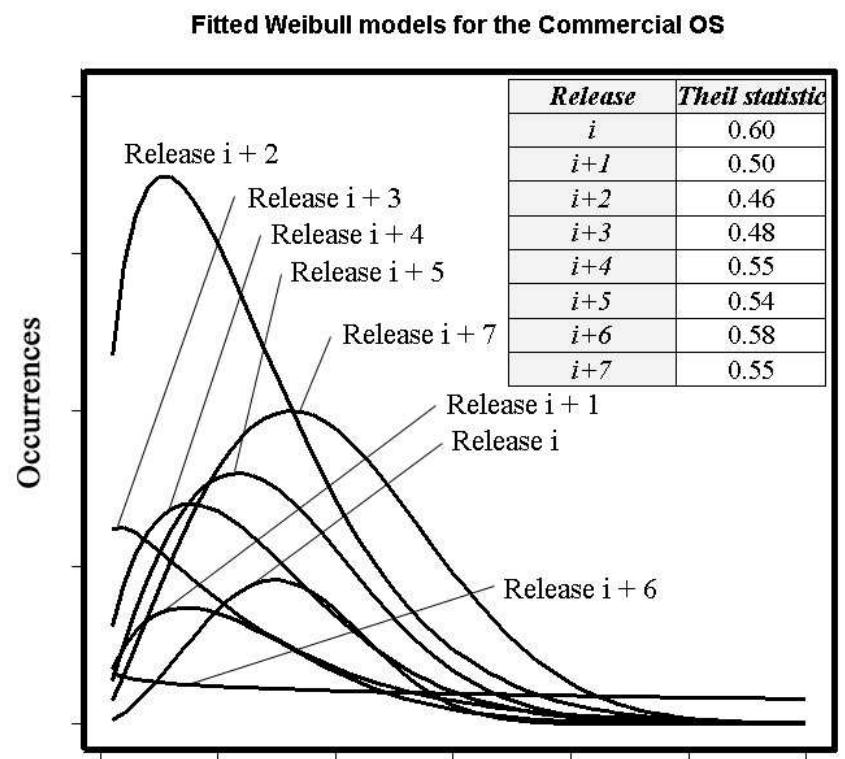

Time

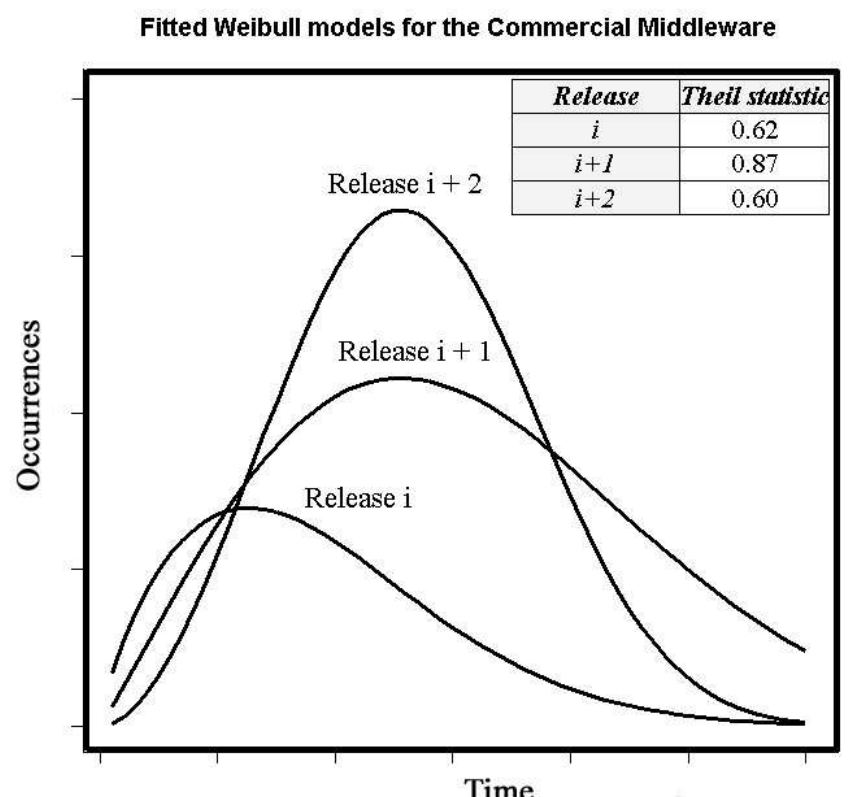

Time
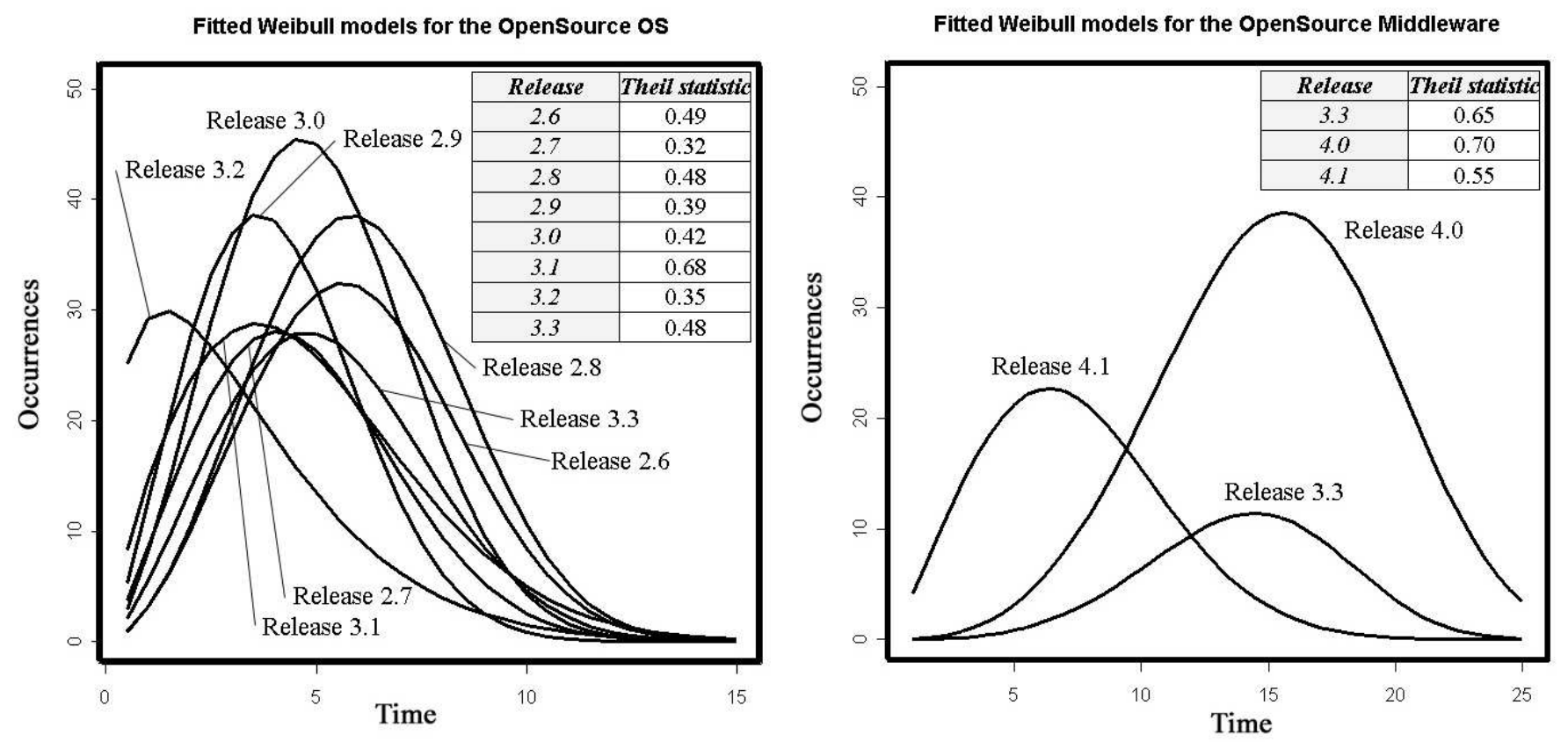
Figure 2. Plots of best-fit Weibull models

We conclude that the Weibull model is the preferred model because the Weibull model is better than other candidate models based on AIC scores in Section 3.3 and is good at projecting defect occurrences based on Theil statistics. Further validation is in [Appendix F].

\section{NAÏVE MODEL PARAMETER EXTRAPOLATION METHODS}

Section 3.4 establishes the Weibull model as the preferred model. However, in order to project defect occurrences we still need to extrapolate model parameters for each new release. Naïve parameter-extrapolation methods that do not consider the changes in characteristics described in section 2.3 may extrapolate parameters that result in poor forecasts. In this section, we develop and empirically test the hypothesis that the moving averages and exponential smoothing methods are inadequate.

\subsection{The moving averages method and the exponential smoothing method}

Both the moving averages and exponential methods are well-established time series methods that represent intuitive, reasonable, and fairly common methods for extrapolating model parameters. The moving averages method extrapolates parameters by taking the average of the best-fit model parameters from the previous $k$ releases. Exponential smoothing is similar, except more recent releases are given more weight, since intuitively more recent releases should be better predictors of the current release. Eick et. al. [6] have used a similar method to predict software defect rates for various software modules.

\subsection{Inadequacies of naïve methods}

Naïve parameter-extrapolation methods may not account for variations in defect-occurrence patterns because the methods do not consider changes in content, development process, deployment and usage patterns, and software and hardware configurations in use between releases. For example, consider a major release and a minor release. A major release that implements new functionality can have significant changes in content, production, and usage. New functionality can lead to substantial code changes and additions. If the schedule is fixed or if there is an insufficient number of trained testers then development constraints may cause inadequate testing. Finally, there may be many more adopters if the functionality being implemented is important to the users. A minor release that offers only minimal improvements over a previous release would have none of the abovementioned conditions. We expect defect-occurrence patterns in the two releases to be drastically different. However, naïve parameter-extrapolation methods do not account for the differences.

We hypothesize that the two naïve parameter-extrapolation methods extrapolate model parameters that produce inadequate defect-occurrence projections for multiple releases of WPSSs.

\subsection{Model parameter extrapolation and forecast evaluation}

We evaluate naïve parameter-extrapolation methods by extrapolating model parameters and then examining the Theil forecasting statistics of the projected defect occurrences. Our hypothesis in section 4.2 will be supported if the two naïve parameter-extrapolation methods fail to consistently produce Theil statistics that are less than one and if the Theil statistics do not consistently improve with more data.

We increase the validity of our results by making the simplifying assumption that the total number of defect occurrences is known. Prior research has already shown that two different software releases are likely to have different total numbers of defect occurrences [5][17]. We remove the possible confound by providing estimates of the total number of defects. We show that naïve parameter-extrapolation methods are inadequate even with this simplification. This topic is discussed in detail in [Appendix D].

Tables 6-9 present fitted $\alpha$ and $\beta$ parameters and Figure 2 presents plots of the best-fit Weibull models. We theorize that changes in characteristics described in Section 2.3 cause the variation in the parameter values.

Table 6. Model parameters for commercial OS

\begin{tabular}{|c|c|c|c|c|c|c|c|c|}
\hline Parameter/Release & $\boldsymbol{i}$ & $\boldsymbol{i}+\mathbf{1}$ & $\boldsymbol{i + 2}$ & $\boldsymbol{i + 3}$ & $\boldsymbol{i + 4}$ & $\boldsymbol{i + 5}$ & $\boldsymbol{i + 6}$ & $\boldsymbol{i + 7}$ \\
\hline$\alpha$ & 2.58 & 1.56 & 1.38 & 1.11 & 1.57 & 1.90 & 0.89 & 2.12 \\
\hline$\beta$ & 9.00 & 7.07 & 6.91 & 6.45 & 7.37 & 8.63 & 131.37 & 10.97 \\
\hline
\end{tabular}


Table 7. Model parameters for commercial middleware system

\begin{tabular}{|c|c|c|c|}
\hline Parameter /Release & $\boldsymbol{i}$ & $\boldsymbol{i}+\mathbf{1}$ & 2.05 \\
\hline$\alpha$ & 1.72 & 2.81 & 17.75 \\
\hline$\beta$ & 10.58 & 14.97 & \\
\hline
\end{tabular}

Table 8. Model parameters for open source OS

\begin{tabular}{|c|c|c|c|c|c|c|c|c|}
\hline Parameter /Release & $\mathbf{2 . 6}$ & $\mathbf{2 . 7}$ & $\mathbf{2 . 8}$ & $\mathbf{2 . 9}$ & $\mathbf{3 . 0}$ & $\mathbf{3 . 1}$ & $\mathbf{3 . 2}$ & $\mathbf{3 . 3}$ \\
\hline$\alpha$ & 2.70 & 2.22 & 2.79 & 2.28 & 2.51 & 1.86 & 1.37 & 2.40 \\
\hline$\beta$ & 6.69 & 5.33 & 6.83 & 4.66 & 5.69 & 5.45 & 3.65 & 5.99 \\
\hline
\end{tabular}

Table 9. Model parameters for open source middleware system

\begin{tabular}{|c|c|c|c|}
\hline Parameter/Release & $\mathbf{3 . 3}$ & $\mathbf{4 . 0}$ & $\mathbf{4 . 1}$ \\
\hline$\alpha$ & 4.19 & 3.84 & 2.21 \\
\hline$\beta$ & 15.44 & 16.89 & 8.39 \\
\hline
\end{tabular}

Table 10 and 11 present Theil statistics resulting from using the moving averages and exponential smoothing methods to select the $\alpha$ and $\beta$ model parameters of the Weibull model. The total number of defects is assumed to be given and is approximated by the total number of defects generated by the best-fit Weibull model.

Table 10. Theil forecasting statistics for projections with the moving averages method

\begin{tabular}{|c|c|c|c|c|c|c|c|}
\hline $\begin{array}{c}\text { Releases/ } \\
\text { System }\end{array}$ & one release & two releases & three releases & four releases & five releases & six releases & seven releases \\
\hline Commercial MW R $i+1$ & 2.26 & & & & & & \\
\hline Commercial MW R $i+2$ & 1.00 & 1.11 & & & & & \\
\hline Open source MW R4.0 & 0.94 & & & & & & \\
\hline Open source MW R4.1 & 2.07 & 2.07 & & & & & \\
\hline Commercial OS $R i+1$ & 0.98 & & & & & & \\
\hline Commercial oS $R i+2$ & 0.50 & 0.88 & & & & & \\
\hline Commercial OS $R i+3$ & 0.56 & 0.61 & 0.86 & & & & \\
\hline Commercial OS $R i+4$ & 0.74 & 0.66 & 0.61 & 0.56 & & & \\
\hline Commercial OS R $i+5$ & 0.94 & 1.30 & 1.29 & 1.22 & 0.89 & & \\
\hline Commercial OS $R i+6$ & 7.33 & 7.44 & 7.43 & 7.46 & 7.52 & 7.63 & \\
\hline Commercial OS $R i+7$ & 3.67 & 3.76 & 3.54 & 3.21 & 2.98 & 2.79 & 2.74 \\
\hline Open source OS R2.7 & 1.13 & & & & & & \\
\hline Open source OS R2.8 & 1.06 & 0.70 & & & & & \\
\hline Open source OS R2.9 & 1.32 & 0.93 & 1.04 & & & & \\
\hline Open source OS R3.0 & 0.87 & 0.42 & 0.43 & 0.44 & & & \\
\hline Open source OS R3.1 & 0.72 & 0.70 & 0.73 & 0.71 & 0.73 & & \\
\hline Open source OS R3.2 & 0.76 & 0.91 & 0.87 & 0.99 & 0.97 & 1.02 & \\
\hline Open source OS R3.3 & 1.56 & 1.10 & 0.85 & 0.86 & 0.66 & 0.66 & 0.57 \\
\hline
\end{tabular}


Table 11. Theil forecasting statistics for projections with the exponential smoothing method

\begin{tabular}{|c|c|c|c|c|c|c|c|}
\hline $\begin{array}{c}\text { Releases/ } \\
\text { System }\end{array}$ & one release & two releases & three releases & four releases & five releases & six releases & seven releases \\
\hline Commercial MW R $i+1$ & 2.26 & & & & & & \\
\hline Commercial MW R $i+2$ & 1.00 & 1.05 & & & & & \\
\hline Open source MW R4.0 & 0.94 & & & & & & \\
\hline Open source MW R4.1 & 2.07 & 2.07 & & & & & \\
\hline Commercial oS $R i+1$ & 0.98 & & & & & & \\
\hline Commercial OS R $i+2$ & 0.50 & 0.81 & & & & & \\
\hline Commercial OS $R i+3$ & 0.56 & 0.60 & 0.77 & & & & \\
\hline Commercial OS $R i+4$ & 0.74 & 0.67 & 0.63 & 0.57 & & & \\
\hline Commercial OS $R i+5$ & 0.94 & 1.24 & 1.24 & 1.21 & 1.06 & & \\
\hline Commercial OS $R i+6$ & 7.33 & 7.42 & 7.41 & 7.43 & 7.46 & 7.50 & \\
\hline Commercial OS R $i+7$ & 3.67 & 3.77 & 3.68 & 3.56 & 3.50 & 3.47 & 3.48 \\
\hline Open source OS R2.7 & 1.13 & & & & & & \\
\hline Open source OS R2.8 & 1.06 & 0.76 & & & & & \\
\hline Open source OS R2.9 & 1.32 & 1.00 & 1.06 & & & & \\
\hline Open source OS R3.0 & 0.87 & 0.43 & 0.44 & 0.42 & & & \\
\hline Open source OS R3.1 & 0.72 & 0.70 & 0.72 & 0.71 & 0.72 & & \\
\hline Open source OS R3.2 & 0.76 & 0.88 & 0.86 & 0.93 & 0.93 & 0.95 & \\
\hline Open source OS R3.3 & 1.56 & 1.18 & 0.99 & 0.98 & 0.87 & 0.86 & 0.82 \\
\hline
\end{tabular}

We evaluate the benefits of including more historical information for naïve parameter-extrapolation methods by comparing the Theil statistics produced using multiple releases against the Theil statistics produced using model parameters of the most recent release as the model parameters for a new release. Improvements are possible since Theil statistics in tables 10 and 11 indicate that in 9 out of 18 or $50 \%$ of the forecasting experiments, using model parameters from the most recent release result in projections that are no better than nochange forecasts. (The exponential smoothing and moving averages methods are identical when extrapolating parameters using only data from the most recent release, thus their results are the same.) However, results show that projections do not improve if we incorporate additional historical information. There are 88 total forecasting experiments for the two naïve parameter-extrapolation methods using data from two or more releases. In 44 out of 88 or $50 \%$ of the forecasting experiments, Theil statistics show no improvement over values from column one release (shaded cells in tables 10 and 11 highlight values showing no improvement). Incorporating additional historical information failed to improve Theil statistics.

Not only do naïve parameter-extrapolation methods fail to improve forecasts with additional data, they produce poor projections overall. Theil statistics are greater than or equal to one in 39 out of 88 or $44 \%$ of the forecasting experiments. Moving averages produced poor projections in $43 \%$ of the forecasting experiments. Exponential smoothing produced poor projections in $45 \%$ of the forecasting experiments. Similar results are produced when we use the naïve parameter-extrapolation methods to extrapolated model parameters for the Gamma model. The details are in [Appendix F]. 
We conclude that there is strong empirical evidence that the Weibull model is the preferred model for modeling defect-occurrence patterns of WPSSs across multiple releases and that the naïve parameterextrapolation methods, moving averages and exponential smoothing, are inadequate in extrapolating model parameters of the Weibull model for defect-occurrence projection.

\section{VALIDATION AND FUTURE WORK}

Our research aims to deal with the real world consequences of defect occurrences in WPSSs. Maintenance planning and software insurance are two methods that can deal with the consequences [13], and both need accurate defect-occurrence projections. This paper set out to address two questions that are important for defectoccurrence projection: is there a type of defect model that provides a good fit to defect-occurrence patterns across multiple releases and in many organizations, and how can model parameters for a new release be extrapolated using historical information.

We have examined historically effective defect-occurrence models. There is extensive defect-occurrence modeling research history to support our belief that our collection of models is well-suited for modeling software defect-occurrence patterns. Despite these efforts, it is possible that a better defect model exists, and we strongly encourage others to replicate our approach using a wider array of models.

The difference in definition of defect occurrences and the time interval used between the software systems strengthens our finding that the Weibull model is the preferred model, since despite the differences in definition, the Weibull model still proved superior. There may be other meaningful ways of counting defects where the Weibull model does not perform as well. Only future research can address this issue.

We have attempted to establish the external validity by including two different types of software systems (middleware and operating systems) developed with two different development styles (commercial and open source). It is not yet clear how adequately this sample represents the population of WPSSs. We regard this as a promising start, but future research should sample additional parts of the WPSS space.

The goal of our research is to develop a defect-occurrence projection method that produces defect-occurrence projections that are better than post-facto fits. Our results show that naïve parameter-extrapolation methods are never better than the post-facto, best-fit, Weibull model (Theil statistics in Figure 2 and Tables 10 and 11). However, a post-facto fit chooses model parameters that minimize the residual error for all data points simultaneously, which does not necessarily produce the best model parameters for each data point. It may be possible to produce better projections than the post-facto fit by updating a-priori projections as more information becomes available, such as after the arrival of field defect-occurrence data or after the release of software patches.

Our results in this paper indicate that the Weibull model is the preferred model for modeling defectoccurrence patterns for multiple releases of WPSSs and that the naïve parameter-extrapolation methods are inadequate. We have claimed that we expected the naïve parameter-extrapolation methods to fail because they do not account for differences in content, development process, deployment and usage patterns, and software and hardware configurations in use. The next step should be to improve parameter-extrapolation methods by predicting the effects on model parameters resulting from changes in characteristics of widely-deployed, production, software systems.

\section{ACKNOWLEDGEMENTS}

This research was supported by the National Science Foundation under Grand CCR-0086003, by the Sloan Software Industry Center at Carnegie Mellon University, and by the NASA High Dependability Computing Program under cooperative agreement NCC-2-1298. Thanks to IBM for making this work possible, Larry Wasserman for his guidance, and Audris Mockus for his insights.

\section{REFERENCES}

[1] K. Bassin and P. Santhanam. Use of software triggers to evaluate software process effectiveness and capture customer usage profiles. In Eighth International Symposium on Software Reliability Engineering, Case Studies, p103-114, 1997.

[2] B. Boehm and et. al. Cost models for future software life cycle processes: COCOMO 2.0. In Annals of Software Engineering Special Volume on Software Process and Product Measurement, Chapter 1,1995. 
[3] M. Buckley and R. Chillarege. Discovering relationships between service and customer satisfaction. In The International Conference on Software Maintenance, p192-200 1995.

[4] R. Chillarege, S. Biyani, and J. Rosenthal. Measurement of failure rate in widely distributed software. In Twenty-Fifth International Symposium on Fault-Tolerant Computing, p424-433, 1995.

[5] S. Chulani. COQUALMO (constructive quality model) a software defect density prediction model. In Project Control for Software Quality, 1999.

[6] S. G. Eick, T. L. Graves, A. F. Karr, J. Marron, and A. Mockus. Does code decay? assessing the evidence from change management data. In IEEE Transactions on Software Engineering, Volume 27, p1-12, 2001.

[7] D.E. Harter, M.S. Krishnan, S.A. Slaughter. Effects of process maturity on quality, cycle time, and effort in software product development. Management Science, Volume 46, p451-466, 2000.

[8] Jakarta Tomcat. http://jakarta.apache.org/tomcat/index.html

[9] W. Jones. Reliability models for very large software systems in industry. In International Symposium Software Reliability Engineering, p17-18, 1991.

[10] W. Jones, J. Hudepohl, T. M. Khoshgoftaar, and E. B. Allen. Application of a usage profile in software quality models. In Third European Conference on Software Maintenance and Reengineering, p148-157, 1999.

[11] G. Kenny. Estimating defects in commercial software during operational use. In IEEE Transactions on Reliability, Volume 42, p107115, 1993.

[12] M. Lehman and L. Belady. Program Evolution: Processes of Software Change. Academic Press, USA, 1985.

[13] P. Li, M. Shaw, and J. Herbsleb. Selecting a defect prediction model for maintenance resource planning and software insurance. In EDSER-5 affiliated with ICSE, p32-37, 2003.

[14] P. Li, M. Shaw, K. Stolarick, and K. Wallnau. The potential for synergy between certification and insurance. In International Workshop on Reuse Economics in conjunction with ICSR, 2002.

[15] P.Li, M.Shaw, J.Herbsleb, B. Ray, and P.Santhanam. Empirical evaluation of defect projection models for widely-deployed production software systems. In CMU tech report CMU-ISRI-04-130, 2004

[16] M. R. Lyu. Handbook of Software Reliability Engineering. IEEE Society Press, USA, 1996.

[17] A. Mockus, D. Weiss, and P. Zhang. Understanding and predicting effort in software projects. In ICSE, p274-284, 2003.

[18] P. Mora and Z. Jelinski. Final report on software reliability study. In McDonell Douglas Astronautic Company Report Number 63921, 1972.

[19] J. Musa. A theory of software reliability and its applications. In IEEE Transaction on Software Engineering, Volume 3, p312-327, 1975.

[20] J. Musa. Operational profiles in software reliability engineering. In IEEE Software, Volume 10, p14-32, 1993.

[21] OpenBSD. http://www.openbsd.org

[22] T. Ostrand and E. Weyuker. The distribution of faults in a large industrial software system. In ISSTA, p55-64, 2002

[23] K.Popstajanova and K. Trivedi. Architecture based approach to reliability assessment of software systems. Performance Evaluation, Volume 45, 2001.

[24] R project for statistical computing. http://www.r-project.org

[25] E. Raymond. Cathedral and the Bazaar. O'Reily \& Associates, USA, 1999.

[26] H. Theil. Applied Economic Forecasting. North-Holland Publishing Company, Netherlands, 1966.

[27] W. Venables and B. Ripley. Modern Applied Statistics with S-plus. Springer Verlag, USA, 1996.

[28] J. Voas. User participation-based software certification. In Eurovav, p267-276, 1999.

[29] A. Wood. Predicting software reliability. In IEEE Computer, Volume 9, p69-77, 1999. 


\section{APPENDIX A. FITTED CANDIDATE MODELS FOR ALL RELEASES}

This section presents fitted candidate models and residual standard errors of the 22 releases of WPSS. The residual standard error is the average error of fitted values for a given model. The NLS model-fitting algorithm uses the residual standard error to fit model parameters. However, the residual standard error does not explicitly penalize models with more parameters to offset their advantage in comparisons. Therefore, to compare candidate models we use the AIC model selection criterion. Some models cannot be fitted for a given release (see Appendix B). Their model curves and residual standard errors will be missing.

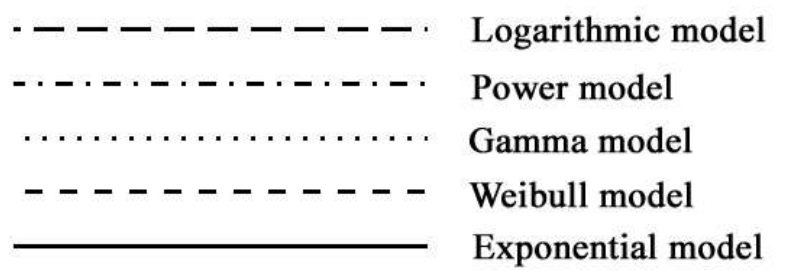

Figure A1. Legend for the candidate models

\section{A1. Fitted candidate models for commercial OS}

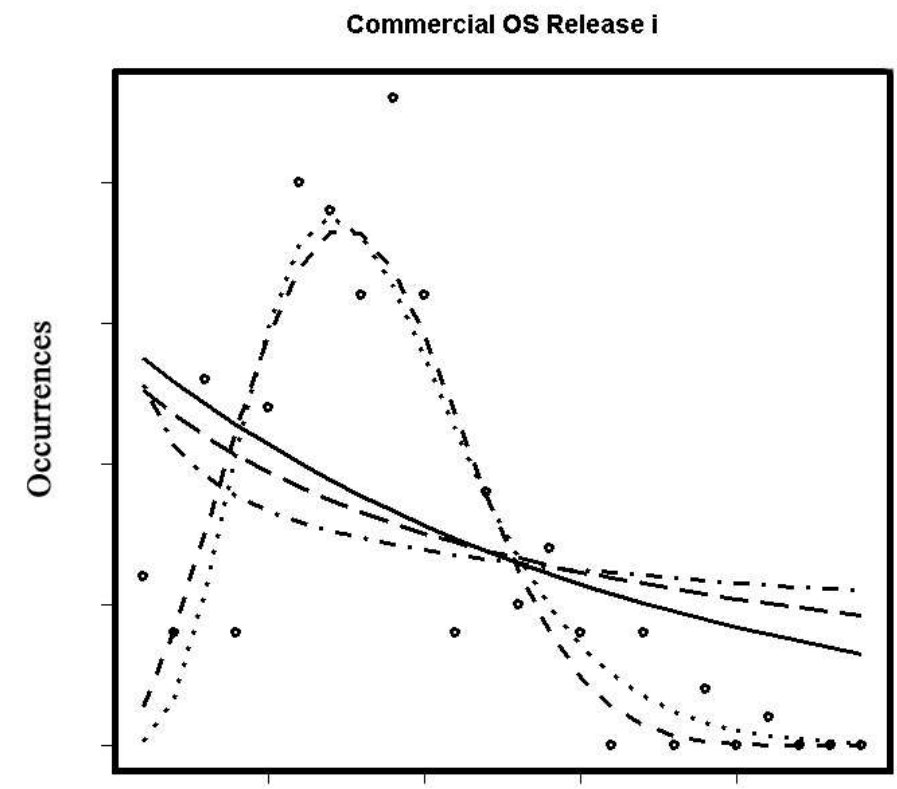

Time

Figure A2. Fitted candidate models for commercial OS Release i

Table A1. Residual standard error for fitted candidate models for commercial OS

\begin{tabular}{|c|c|c|c|c|c|}
\hline Model/Release & Exponential model & Weibull model & Gamma model & Power model & Logarithmic model \\
\hline $\begin{array}{c}\text { Commercial OS } R \\
i\end{array}$ & 6.26 on $22 \mathrm{df}$ & 3.47 on $21 \mathrm{df}$ & $3.652 \mathrm{on} 21 \mathrm{df}$ & 7.016 on $22 \mathrm{df}$ & $6.571 \mathrm{on} 22 \mathrm{df}$ \\
\hline
\end{tabular}




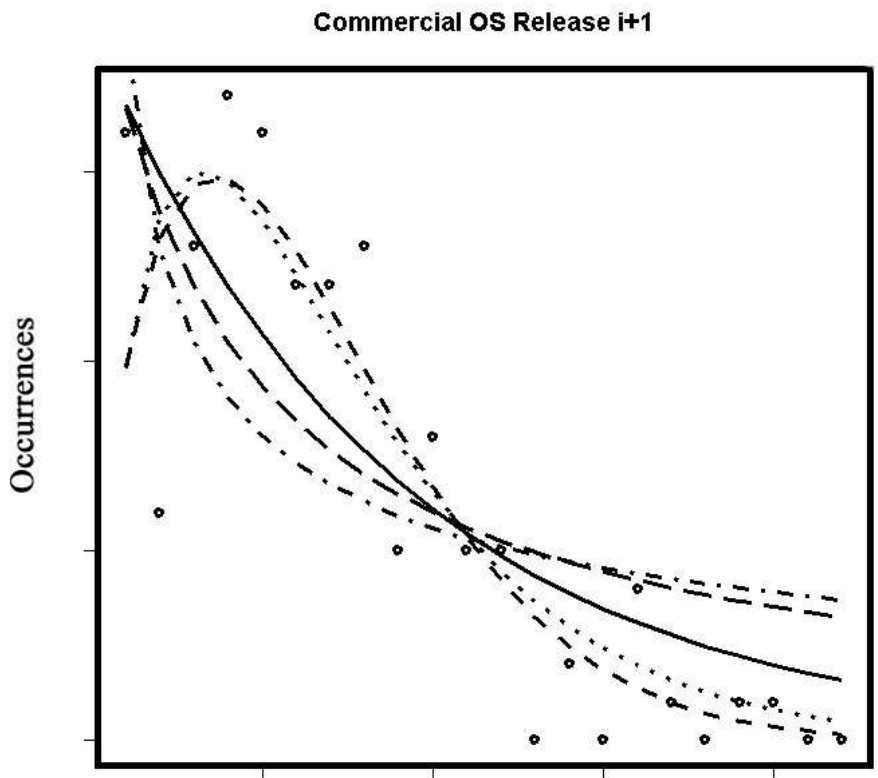

Time

Figure A3. Fitted candidate models for commercial OS Release i+1

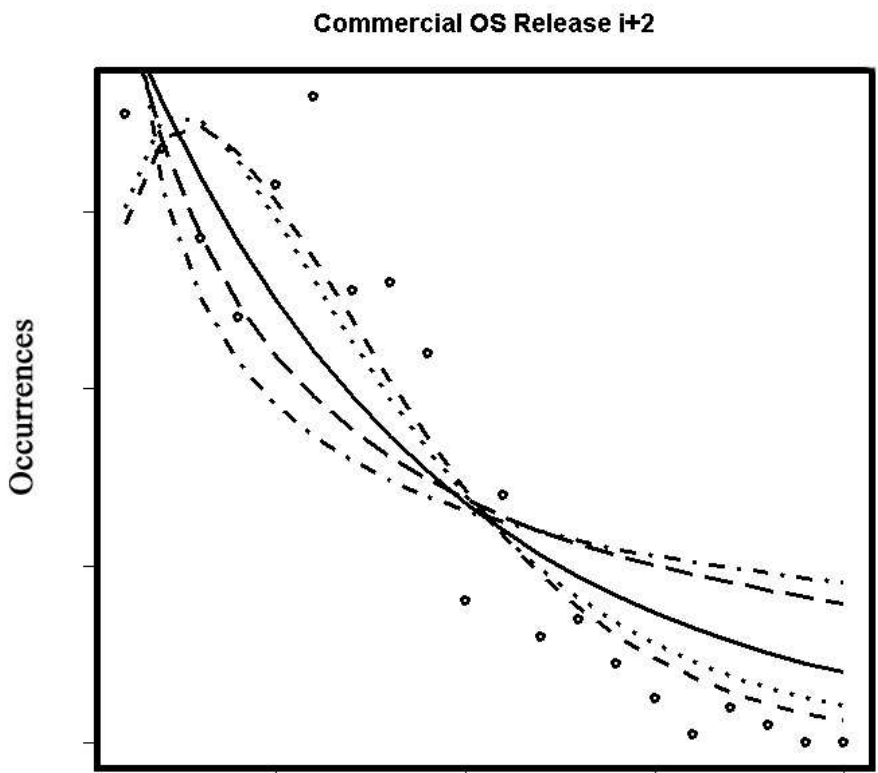

Time

Figure A4. Fitted candidate models for commercial OS Release i+2

Table A2. Residual standard error for fitted candidate models for commercial OS

\begin{tabular}{|c|c|c|c|c|c|}
\hline Model/Release & Exponential model & Weibull model & Gamma model & Power model & Logarithmic model \\
\hline $\begin{array}{c}\text { Commercial OS } R \\
i+1\end{array}$ & 3.475 on $20 \mathrm{df}$ & 2.786 on $19 \mathrm{df}$ & 2.961 on $19 \mathrm{df}$ & $4.554 \mathrm{on} 20 \mathrm{df}$ & $4.102 \mathrm{on} 20 \mathrm{df}$ \\
\hline $\begin{array}{c}\text { Commercial OS } R \\
i+2\end{array}$ & 12.22 on $18 \mathrm{df}$ & 9.576 on $17 \mathrm{df}$ & $10.34 \mathrm{on} 17 \mathrm{df}$ & $18.34 \mathrm{on} 18 \mathrm{df}$ & $15.82 \mathrm{on} 18 \mathrm{df}$ \\
\hline
\end{tabular}




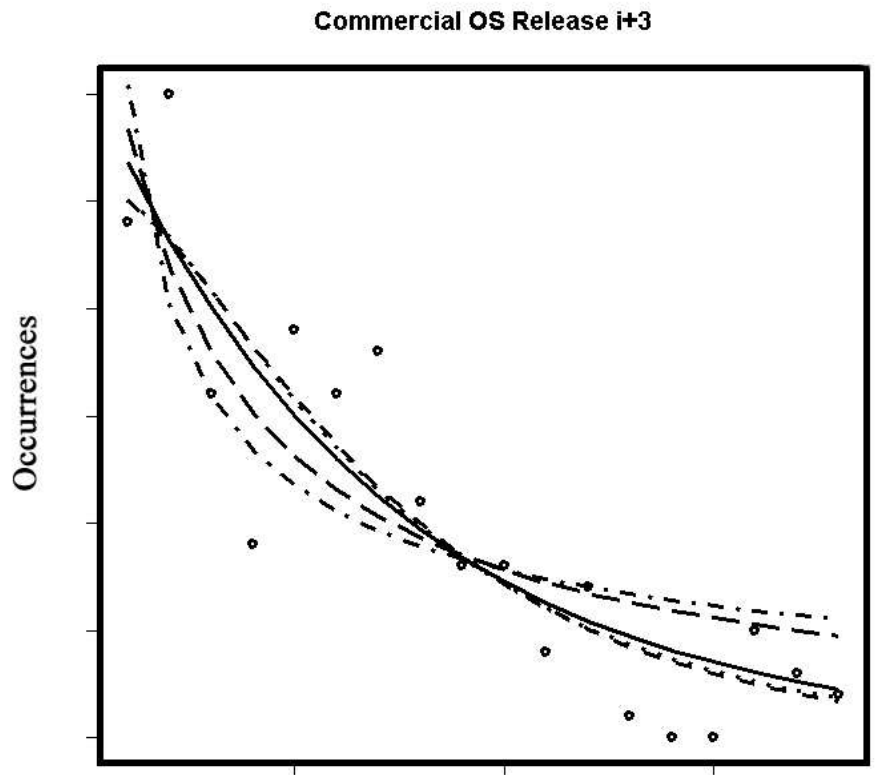

Time

Figure A5. Fitted candidate models for commercial OS Release i+3

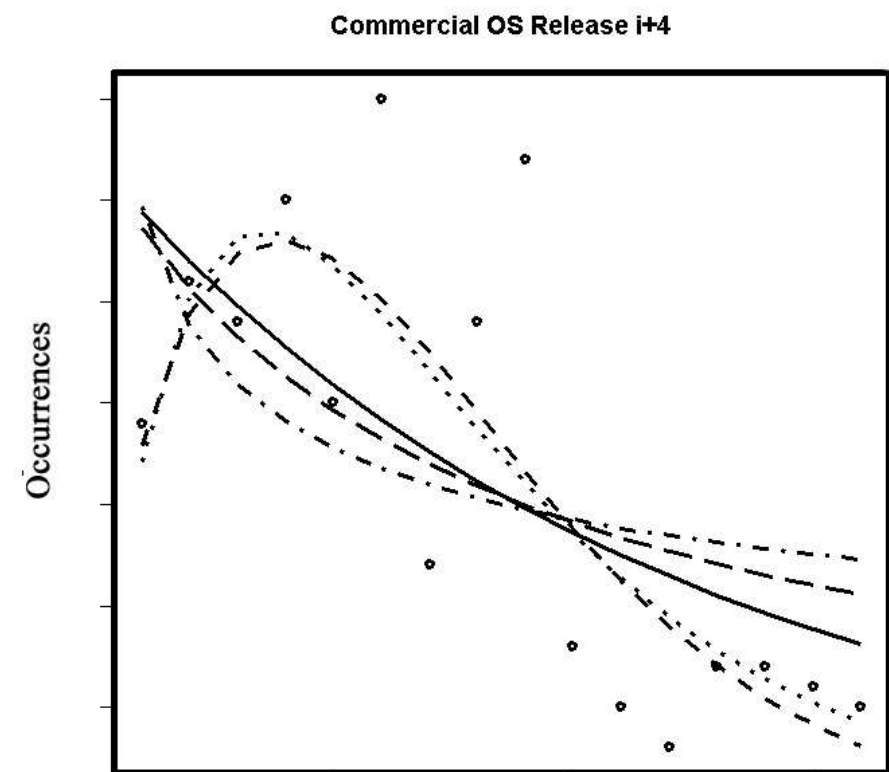

Time

Figure A6. Fitted candidate models for commercial OS Release i+4

Table A3. Residual standard error for fitted candidate models for commercial OS

\begin{tabular}{|c|c|c|c|c|c|}
\hline Model/Release & Exponential model & Weibull model & Gamma model & Power model & Logarithmic model \\
\hline $\begin{array}{c}\text { Commercial OS R } \\
i+3\end{array}$ & $4.093 \mathrm{on} 16 \mathrm{df}$ & 4.161 on $15 \mathrm{df}$ & $4.172 \mathrm{on} 15 \mathrm{df}$ & 5.34 on $16 \mathrm{df}$ & $4.733 \mathrm{on} 16 \mathrm{df}$ \\
\hline $\begin{array}{c}\text { Commercial OS R } \\
i+4\end{array}$ & 8.446 on $14 \mathrm{df}$ & 7.077 on $13 \mathrm{df}$ & $7.198 \mathrm{on} 13 \mathrm{df}$ & $9.881 \mathrm{on} 14 \mathrm{df}$ & $9.001 \mathrm{on} 14 \mathrm{df}$ \\
\hline
\end{tabular}




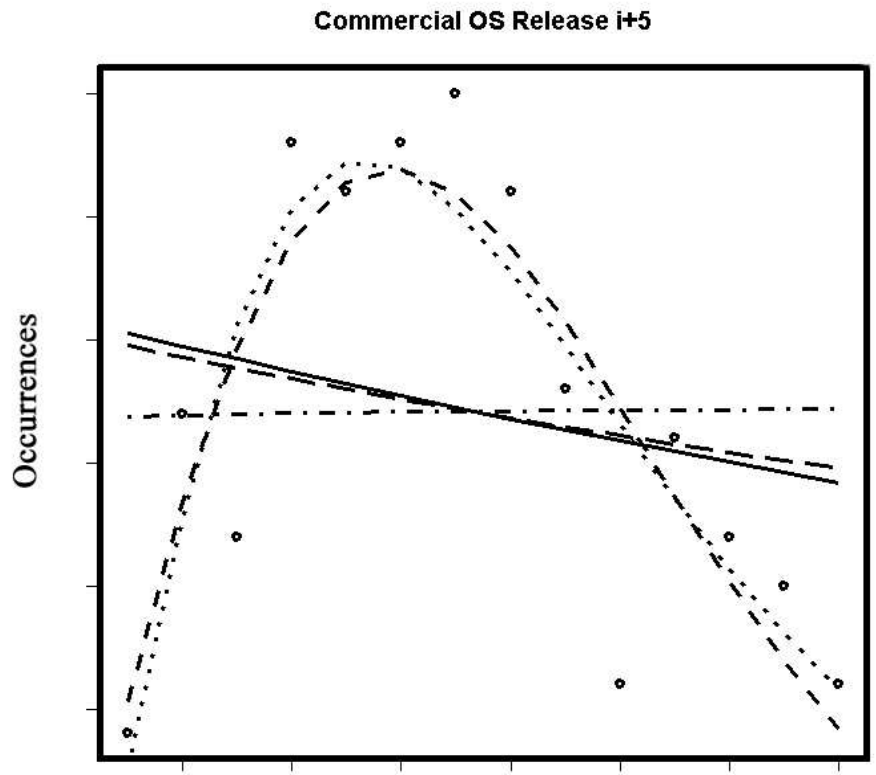

Time

Figure A7. Fitted candidate models for commercial OS Release i+5

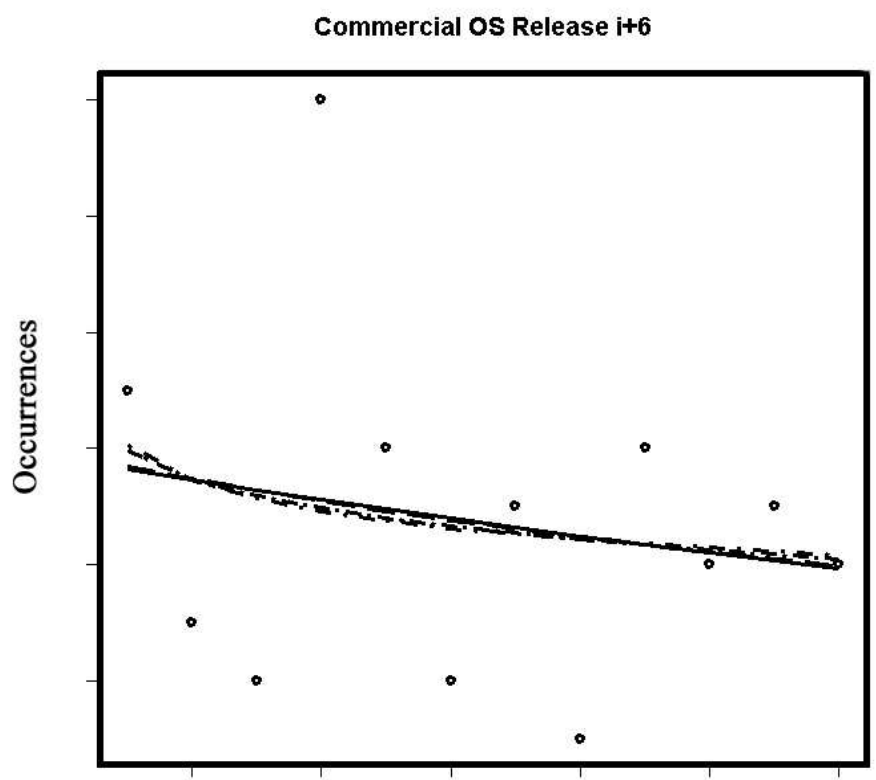

Time

Figure A8. Fitted candidate models for commercial OS Release i+6

Table A4. Residual standard error for fitted candidate models for commercial OS

\begin{tabular}{|c|c|c|c|c|c|}
\hline $\begin{array}{c}\text { Model/Release } \\
\text { Commercial OS R } \\
i+5\end{array}$ & Exponential model & Weibull model & Gamma model & Power model & Logarithmic model \\
\hline $\begin{array}{c}\text { Commercial OS } R \\
i+6\end{array}$ & 3.016 on $10 \mathrm{df}$ & 3.176 on $9 \mathrm{df}$ & 3.176 on $9 \mathrm{df}$ & $3.013 \mathrm{on} 10 \mathrm{df}$ & $3.015 \mathrm{on} 10 \mathrm{df}$ \\
\hline
\end{tabular}




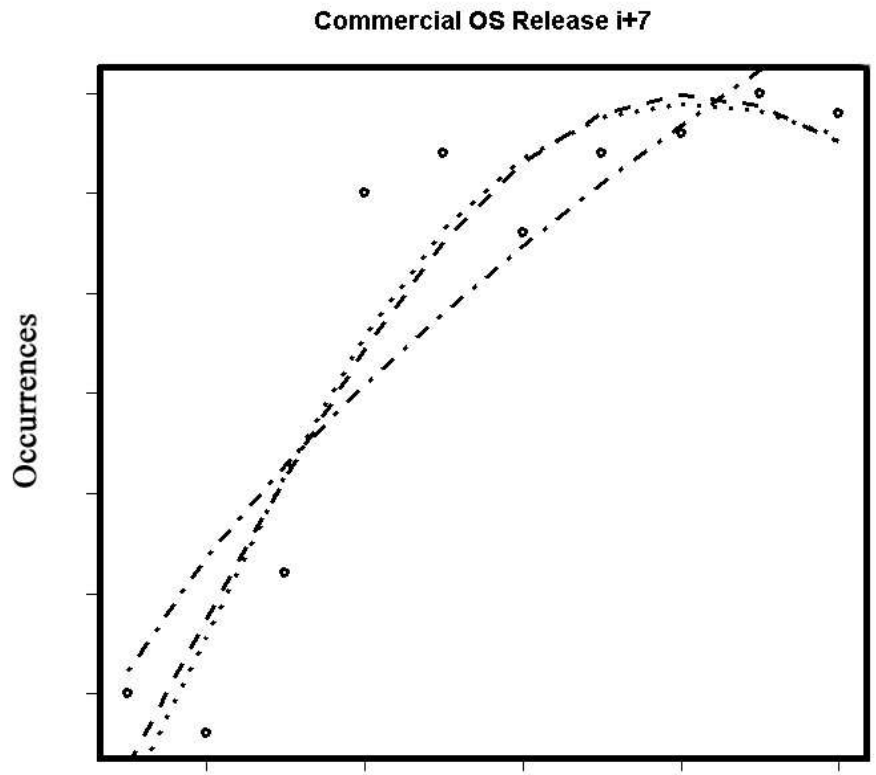

Time

Figure A9. Fitted candidate models for commercial OS Release i+7

Table A5. Residual standard error for fitted candidate models for commercial OS

\begin{tabular}{|c|c|c|c|c|c|}
\hline Model/Release & Exponential model & Weibull model & Gamma model & Power model & Logarithmic model \\
\hline $\begin{array}{c}\text { Commercial OS R } \\
i+7\end{array}$ & INF & 4.943 on $7 \mathrm{df}$ & 4.811 on $7 \mathrm{df}$ & 6.039 on $8 \mathrm{df}$ & Singular Gradient \\
\hline
\end{tabular}




\section{A2. Fitted candidate models for commercial middleware system}

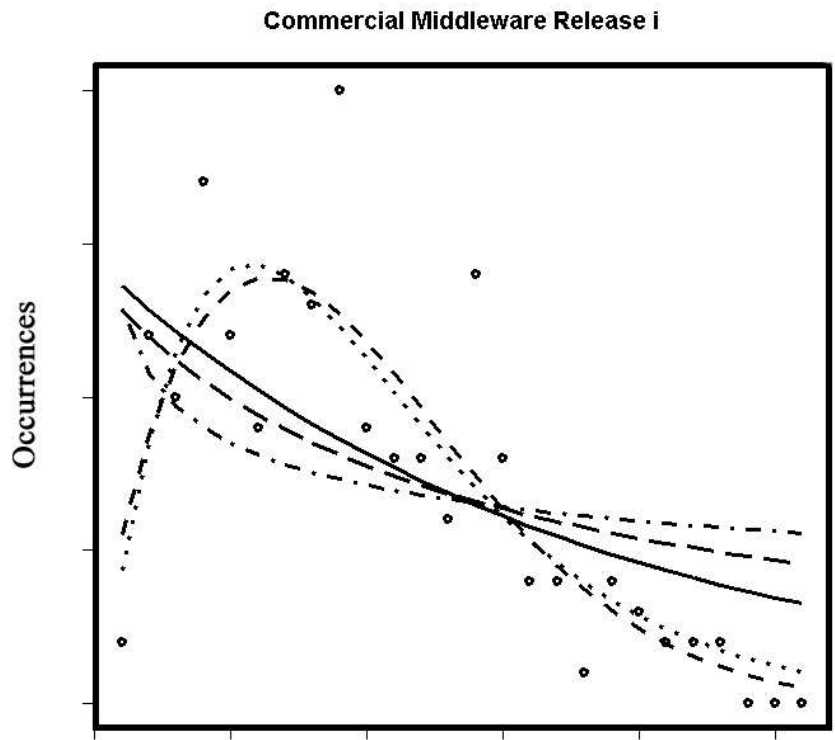

Time

Figure A10. Fitted candidate models for commercial middleware system Release i

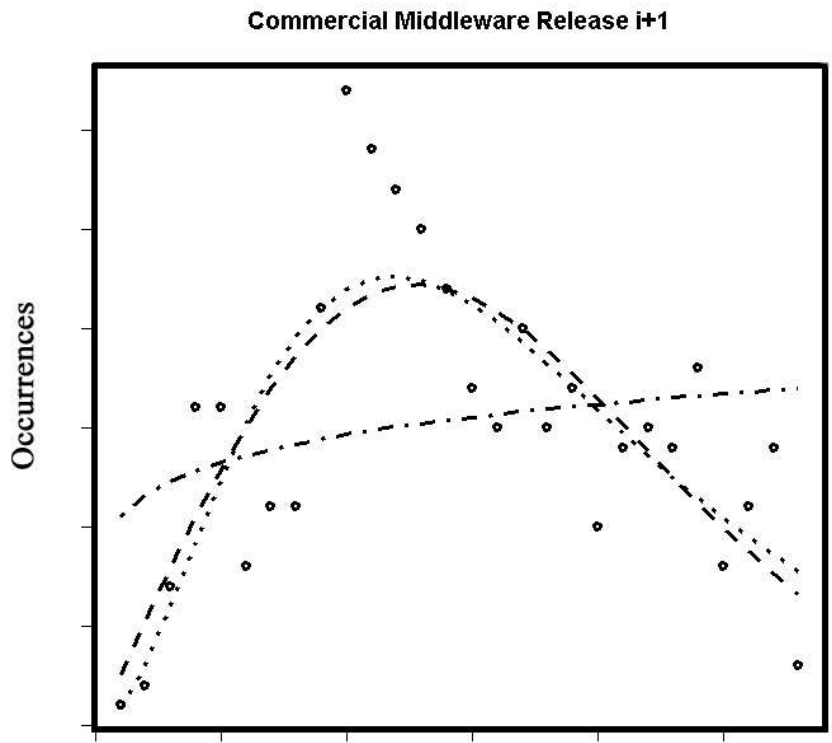

Time

Figure A11. Fitted candidate models for commercial middleware system Release i+1

Table A6. Residual standard error for fitted candidate models for commercial middleware system

\begin{tabular}{|c|c|c|c|c|c|}
\hline Model/Release & Exponential model & Weibull model & Gamma model & Power model & Logarithmic model \\
\hline $\begin{array}{c}\text { Commercial } M W \\
R i\end{array}$ & 4.446 on $24 \mathrm{df}$ & 3.003 on $23 \mathrm{df}$ & $3.014 \mathrm{on} 23 \mathrm{df}$ & 5.317 on $24 \mathrm{df}$ & $4.77 \mathrm{on} 24 \mathrm{df}$ \\
\hline $\begin{array}{c}\text { Commercial } M W \\
R \text { i }+1\end{array}$ & Singular Gradient & 5.023 on $25 \mathrm{df}$ & $4.9 \mathrm{on} 25 \mathrm{df}$ & 7.585 on $26 \mathrm{df}$ & Singular Gradient \\
\hline
\end{tabular}




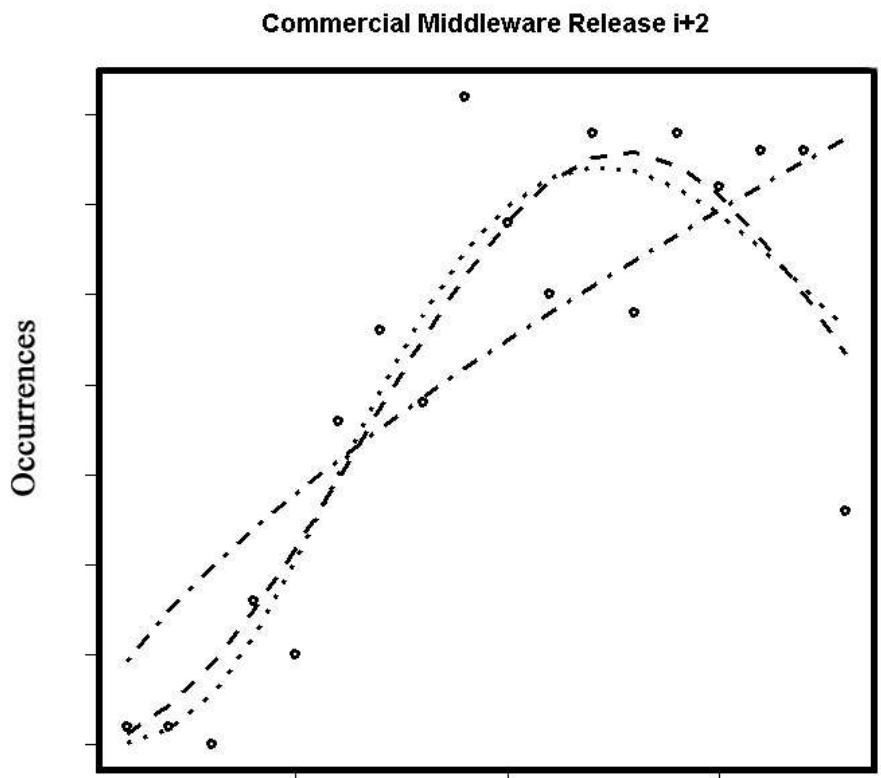

Time

Figure A12. Fitted candidate models for commercial middleware system Release i+2

Table A7. Residual standard error for fitted candidate models for commercial middleware system

\begin{tabular}{|c|c|c|c|c|c|}
\hline Model/Release & Exponential model & Weibull model & Gamma model & Power model & Logarithmic model \\
\hline $\begin{array}{c}\text { Commercial MW } \\
\text { R } i+2\end{array}$ & Singular Gradient & $5.677 \mathrm{on} 15 \mathrm{df}$ & $5.588 \mathrm{on} 15 \mathrm{df}$ & $8.287 \mathrm{on} 16 \mathrm{df}$ & Singular Gradient \\
\hline
\end{tabular}




\section{A3. Fitted candidate models for open source OS}

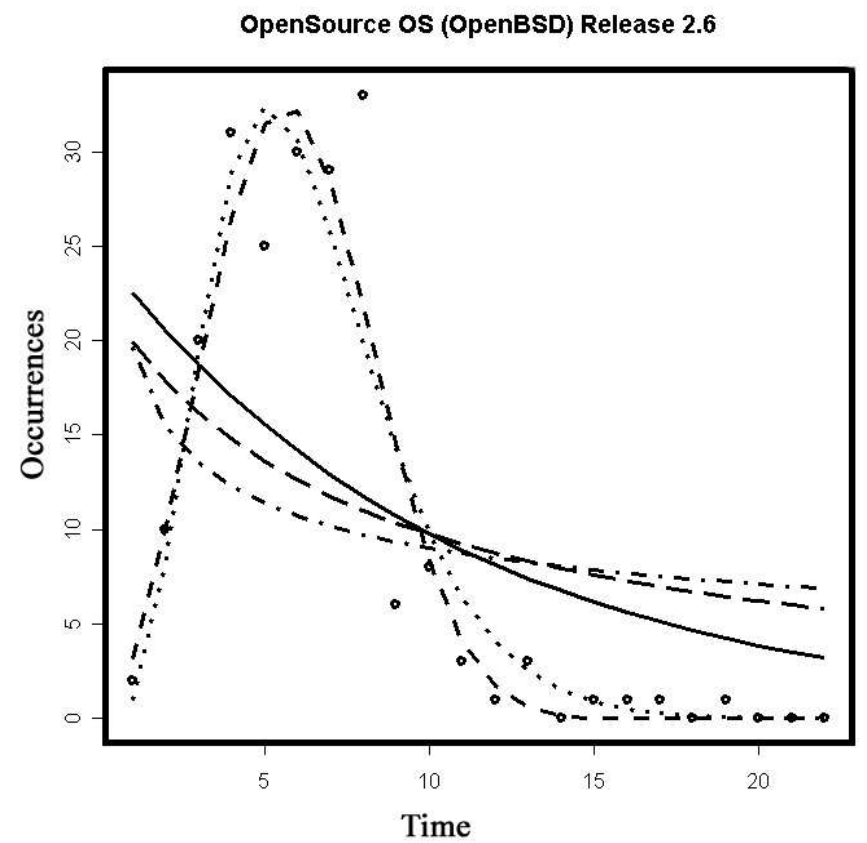

Figure A13. Fitted candidate models for open source OS Release 2.6

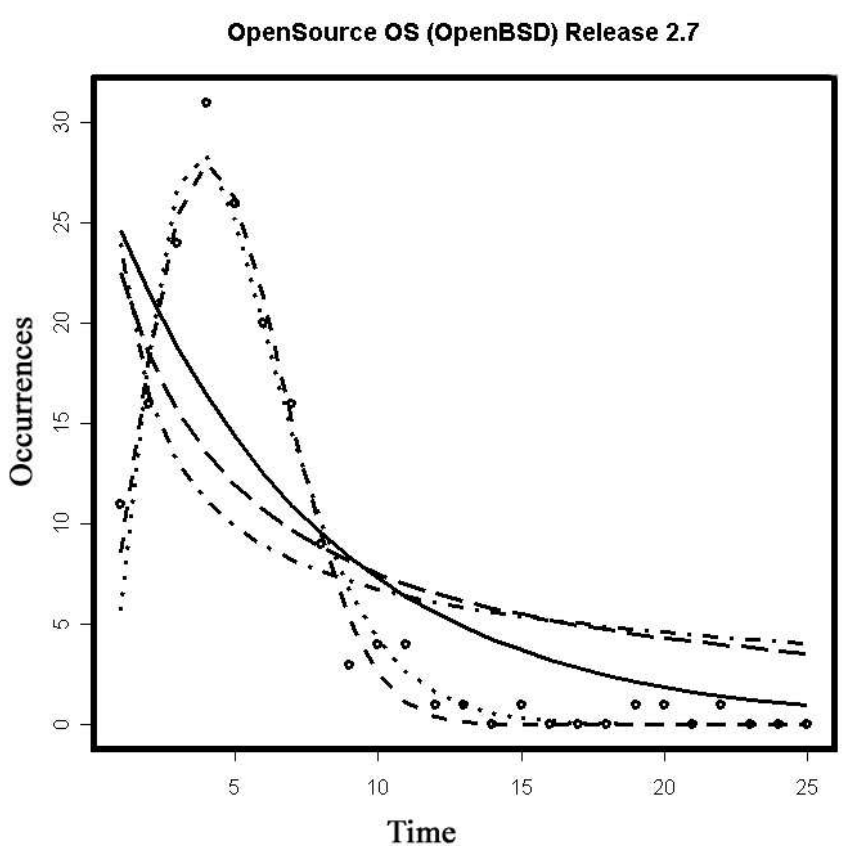

Figure A14. Fitted candidate models for open source OS Release 2.7

Table A8. Residual standard error for fitted candidate models for open source OS

\begin{tabular}{|c|c|c|c|c|c|}
\hline Model/Release & Exponential model & Weibull model & Gamma model & Power model & Logarithmic model \\
\hline $\begin{array}{c}\text { Open source } \\
\text { OS R2.6 }\end{array}$ & 10.22 on $20 \mathrm{df}$ & 3.839 on $19 \mathrm{df}$ & 4.266 on $19 \mathrm{df}$ & 11.74 on $20 \mathrm{df}$ & 10.93 on $20 \mathrm{df}$ \\
\hline $\begin{array}{c}\text { Open source } \\
\text { OS R2.7 }\end{array}$ & 5.946 on $23 \mathrm{df}$ & 1.423 on $22 \mathrm{df}$ & 1.811 on $22 \mathrm{df}$ & $7.92 \mathrm{on} 23 \mathrm{df}$ & 7.109 on $23 \mathrm{df}$ \\
\hline
\end{tabular}




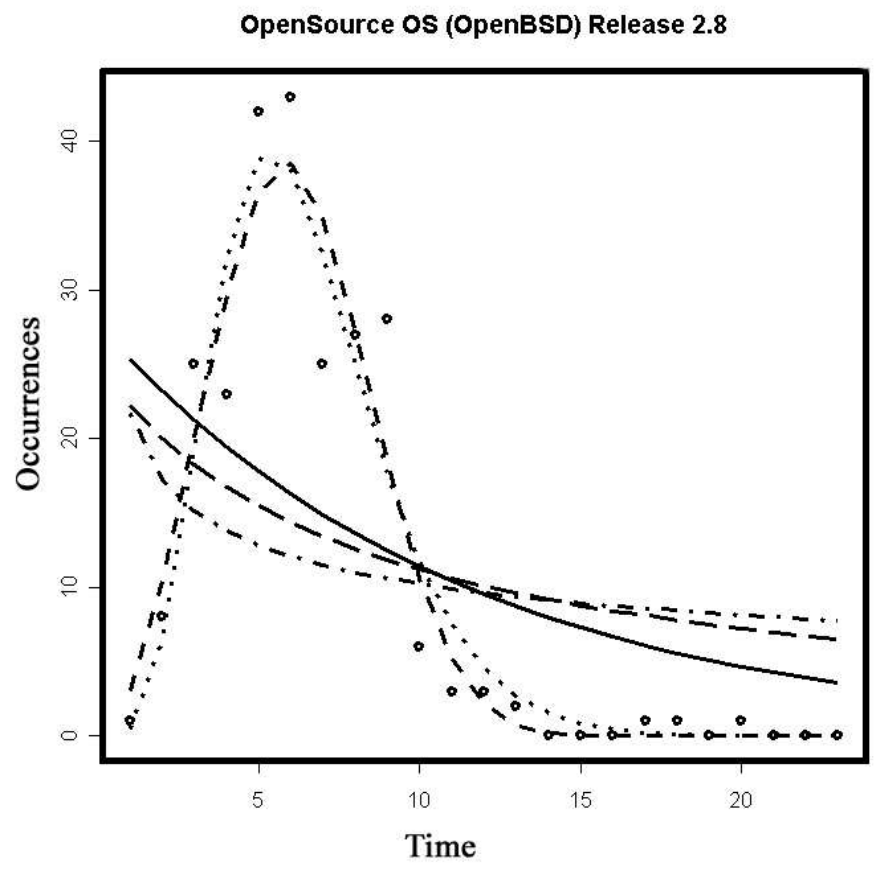

Figure A15. Fitted candidate models for open source OS Release 2.8

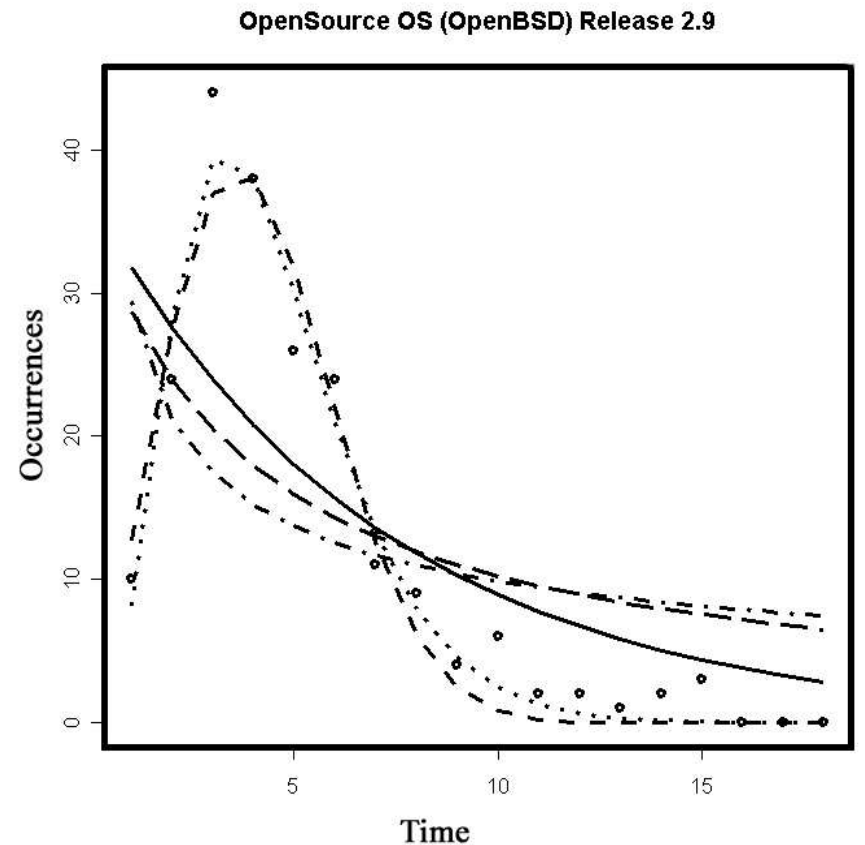

Figure A16. Fitted candidate models for open source OS Release 2.9

Table A9. Residual standard error for fitted candidate models for open source OS

\begin{tabular}{|c|c|c|c|c|c|}
\hline Model/Release & Exponential model & Weibull model & Gamma model & Power model & Logarithmic model \\
\hline $\begin{array}{c}\text { Open source } \\
\text { OS R2.8 }\end{array}$ & 12.24 on $21 \mathrm{df}$ & 4.187 on $20 \mathrm{df}$ & 4.371 on $20 \mathrm{df}$ & $13.92 \mathrm{on} 21 \mathrm{df}$ & $13.02 \mathrm{on} 21 \mathrm{df}$ \\
\hline $\begin{array}{c}\text { Open source } \\
\text { OS R2.9 }\end{array}$ & 9.693 on $16 \mathrm{df}$ & 3.332 on $15 \mathrm{df}$ & 2.607 on $15 \mathrm{df}$ & 12.14 on $16 \mathrm{df}$ & 11 on $16 \mathrm{df}$ \\
\hline
\end{tabular}




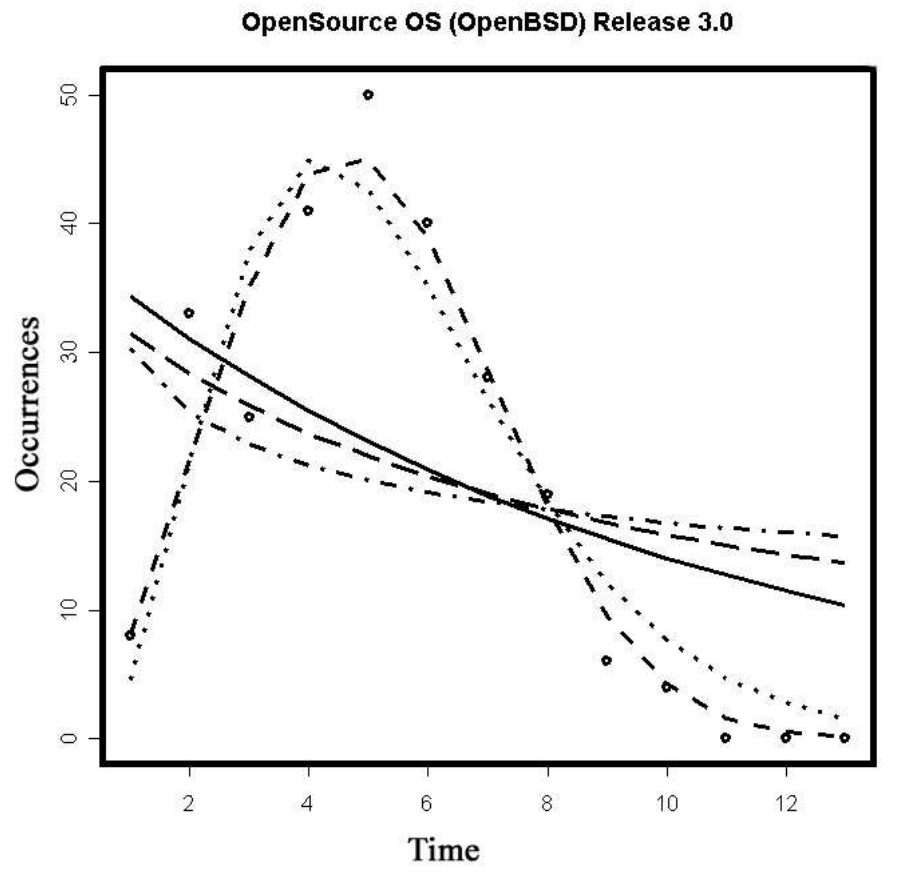

Figure A17. Fitted candidate models for open source OS Release 3.0

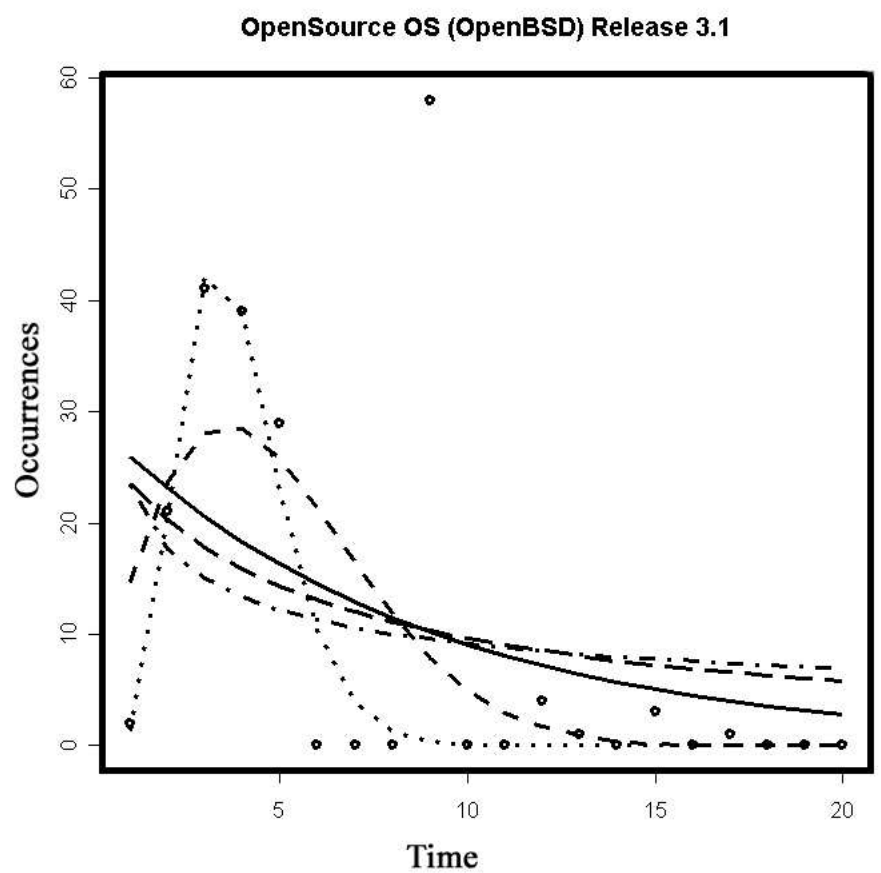

Figure A18. Fitted candidate models for open source OS Release 3.1

Table A10. Residual standard error for fitted candidate models for open source OS

\begin{tabular}{|c|c|c|c|c|c|}
\hline Model/Release & Exponential model & Weibull model & Gamma model & Power model & Logarithmic model \\
\hline $\begin{array}{c}\text { Open source } \\
\text { OS R3.0 }\end{array}$ & 15.72 on $11 \mathrm{df}$ & 5.313 on $10 \mathrm{df}$ & 7.035 on $10 \mathrm{df}$ & 17.68 on $11 \mathrm{df}$ & $16.52 \mathrm{on} 11 \mathrm{df}$ \\
\hline $\begin{array}{c}\text { Open source } \\
\text { OS R3.1 }\end{array}$ & 16.1 on $18 \mathrm{df}$ & 15.1 on $17 \mathrm{df}$ & 14.37 on $17 \mathrm{df}$ & $17.23 \mathrm{on} 18 \mathrm{df}$ & $16.62 \mathrm{on} 18 \mathrm{df}$ \\
\hline
\end{tabular}




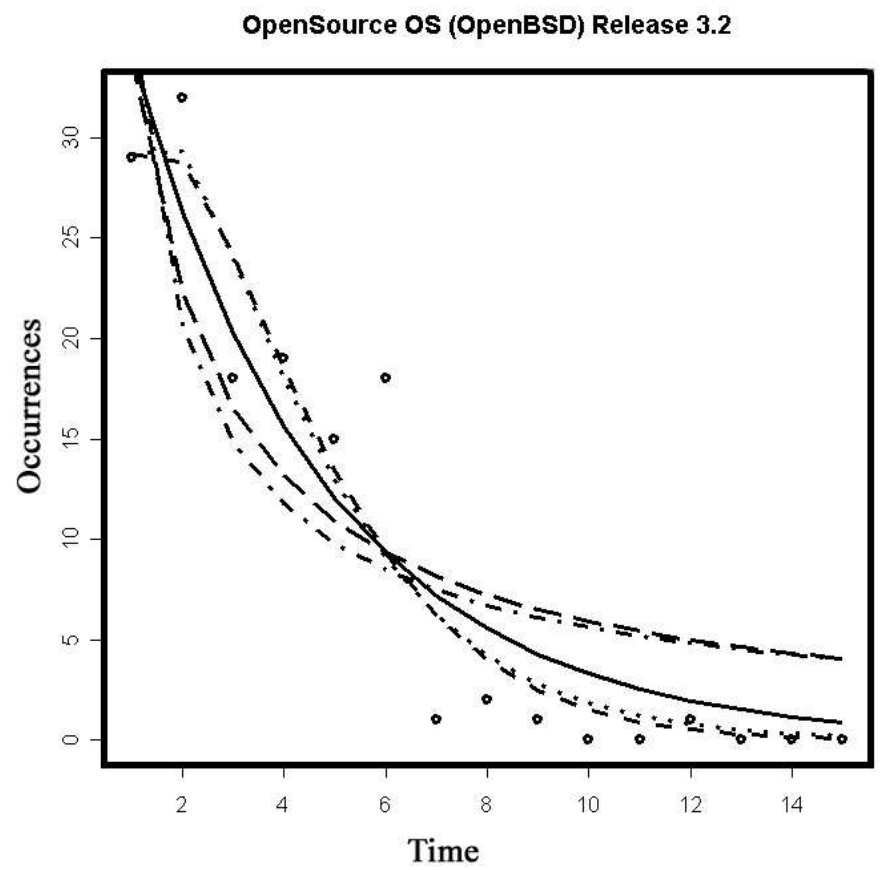

Figure A19. Fitted candidate models for open source OS Release 3.2

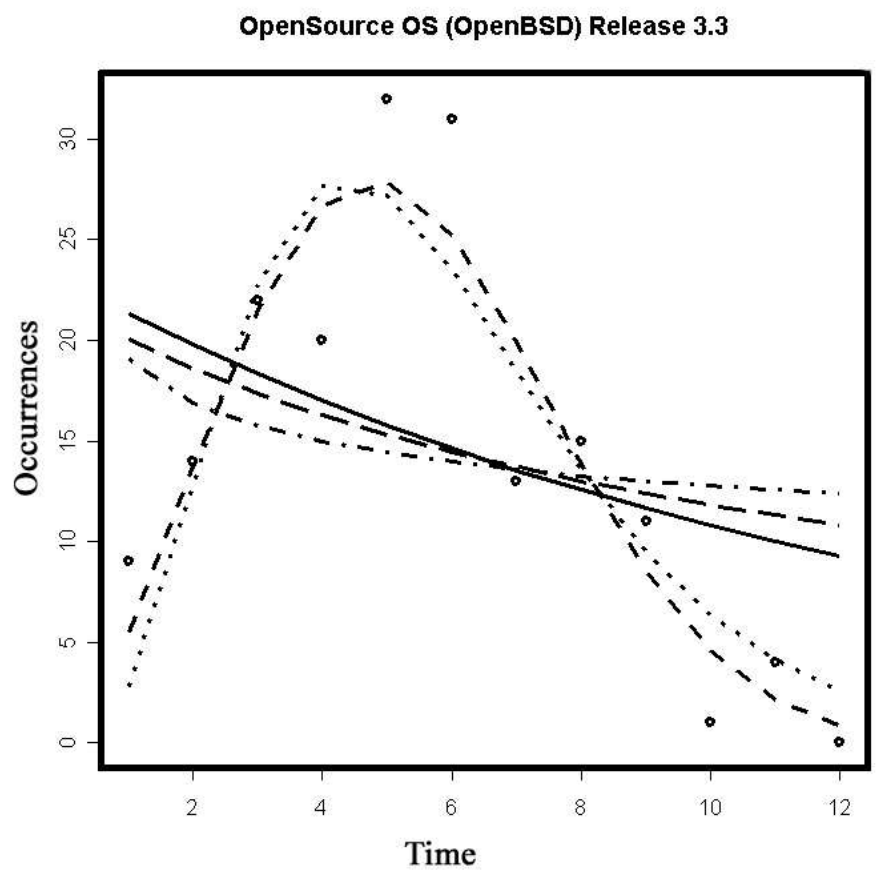

Figure A20. Fitted candidate models for open source OS Release 3.3

Table A11. Residual standard error for fitted candidate models for open source OS

\begin{tabular}{|c|c|c|c|c|c|}
\hline Model/Release & Exponential model & Weibull model & Gamma model & Power model & Logarithmic model \\
\hline $\begin{array}{c}\text { Open source } \\
\text { OS R3.2 }\end{array}$ & 4.325 on $13 \mathrm{df}$ & 3.686 on $12 \mathrm{df}$ & 3.746 on $12 \mathrm{df}$ & $6.671 \mathrm{on} 13 \mathrm{df}$ & $6.163 \mathrm{on} 13 \mathrm{df}$ \\
\hline $\begin{array}{c}\text { Open source } \\
\text { OS R3.3 }\end{array}$ & 9.823 on $10 \mathrm{df}$ & 4.477 on $9 \mathrm{df}$ & 5.252 on $9 \mathrm{df}$ & $10.71 \mathrm{on} 10 \mathrm{df}$ & $10.12 \mathrm{on} 10 \mathrm{df}$ \\
\hline
\end{tabular}




\section{A4. Fitted candidate models for open source middleware System}

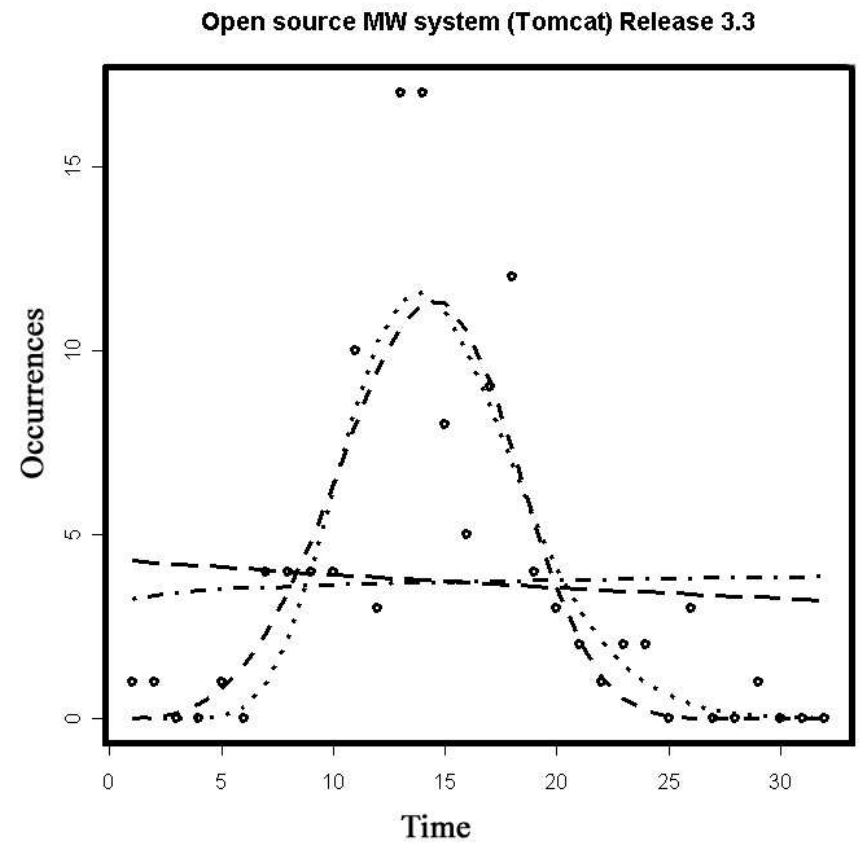

Figure A21. Fitted candidate models for open source middleware Release 3.3

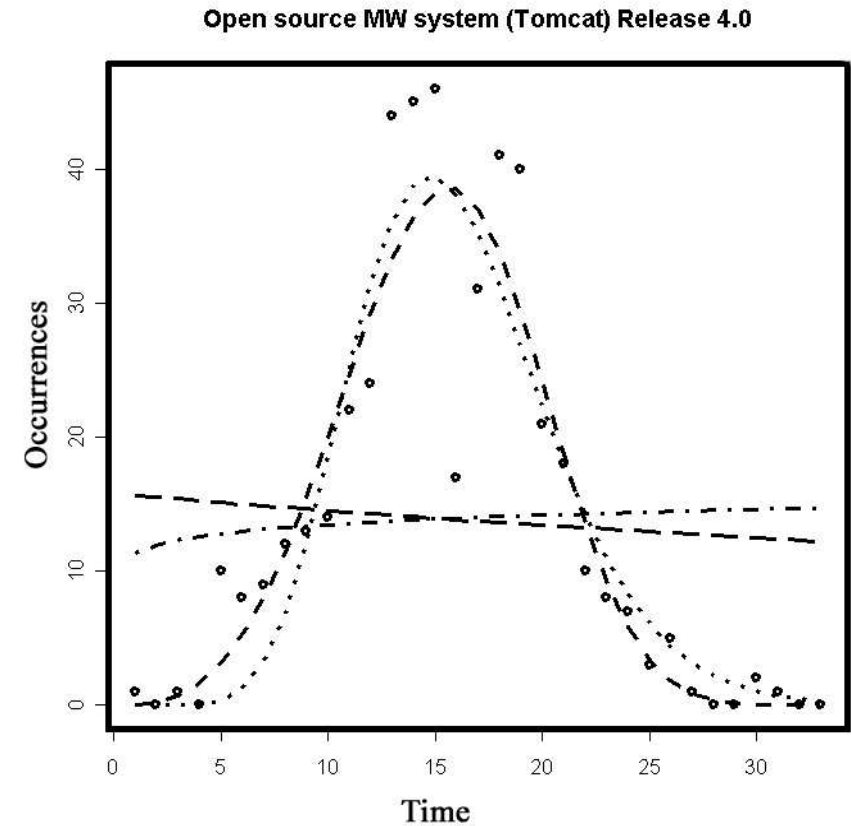

Figure A23. Fitted candidate models for open source middleware Release 4.0

Table A12. Residual standard error for fitted candidate models for open source middleware system

\begin{tabular}{|c|c|c|c|c|c|}
\hline Model/Release & Exponential model & Weibull model & Gamma model & Power model & Logarithmic model \\
\hline $\begin{array}{c}\text { Open source MW } \\
\text { R3.3 }\end{array}$ & INF & 2.711 on $29 \mathrm{df}$ & 2.699 on $29 \mathrm{df}$ & $4.76 \mathrm{on} 30 \mathrm{df}$ & $4.736 \mathrm{on} 30 \mathrm{df}$ \\
\hline $\begin{array}{c}\text { Open source MW } \\
\text { R4.0 }\end{array}$ & INF & 6.014 on $30 \mathrm{df}$ & 6.359 on $30 \mathrm{df}$ & 15.19 on $31 \mathrm{df}$ & $15.15 \mathrm{on} 31 \mathrm{df}$ \\
\hline
\end{tabular}




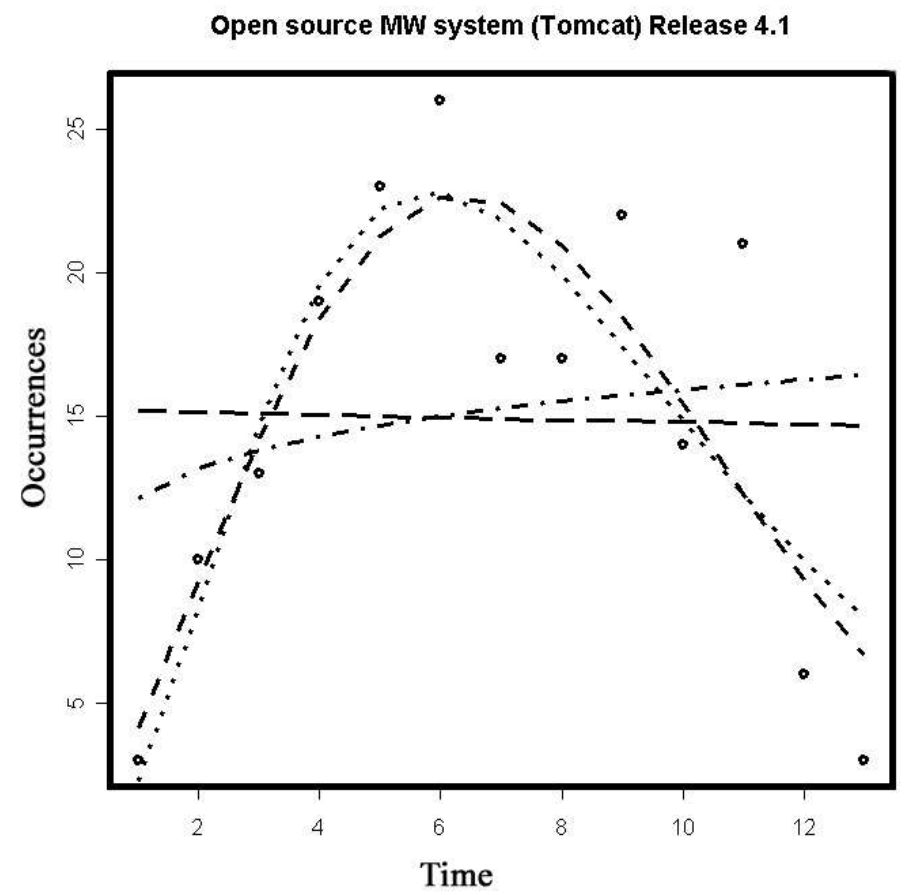

Figure A24. Fitted candidate models for open source middleware Release 4.1

Table A13. Residual standard error for fitted candidate models for open source middleware system

\begin{tabular}{|c|c|c|c|c|c|}
\hline Model/Release & Exponential model & Weibull model & Gamma model & Power model & Logarithmic model \\
\hline $\begin{array}{c}\text { Open source } M W \\
\text { R4.1 }\end{array}$ & INF & 4.223 on $10 \mathrm{df}$ & 4.329 on $10 \mathrm{df}$ & $7.774 \mathrm{on} 11 \mathrm{df}$ & $7.933 \mathrm{on} 11 \mathrm{df}$ \\
\hline
\end{tabular}

\section{APPENDIX B. THE INABILITY TO FIT MODEL PARAMETERS}

A candidate model is inappropriate for modeling defect occurrences of a given release when either the infinity or the singular gradient error occurs. The infinity and singular gradient errors indicate that the model-fitting algorithm is unable to fit model parameters for the model. The releases for which at least one candidate model failed to be fitted are in figure B1. We provide an intuitive explanation of why candidate models that fail to be fitted are inappropriate for modeling defect occurrences by examining the relationship between the parameterizations of the models and the data. 


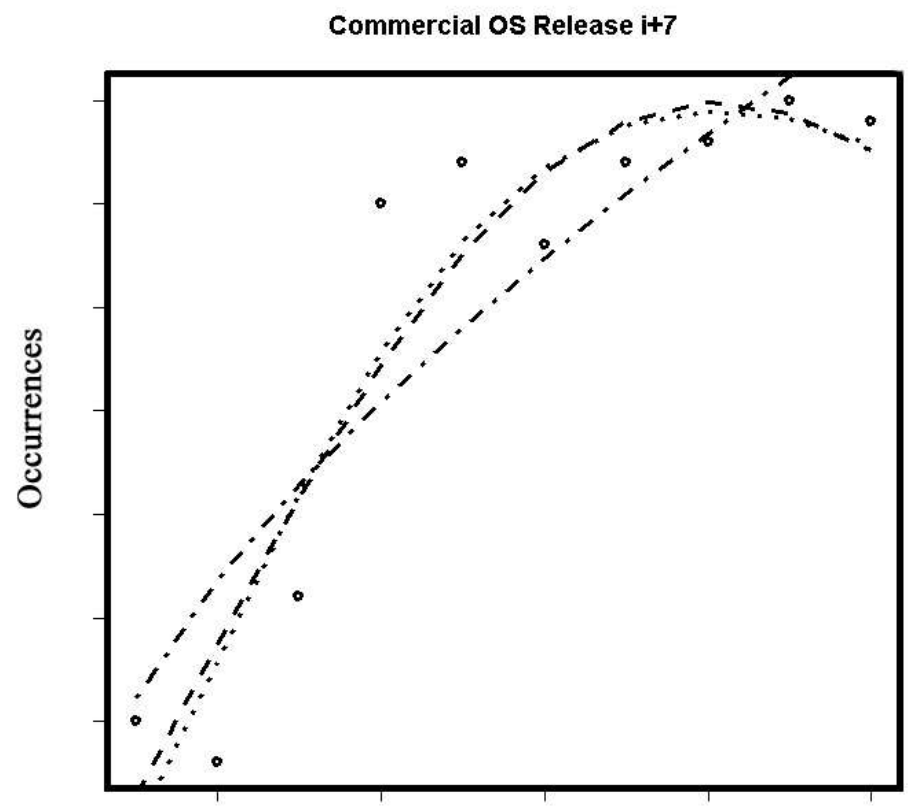

Time

Figure B1. Commercial OS Release i+7, which failed to fit the Exponential and Logarithmic models

Commercial Middleware Release i+1

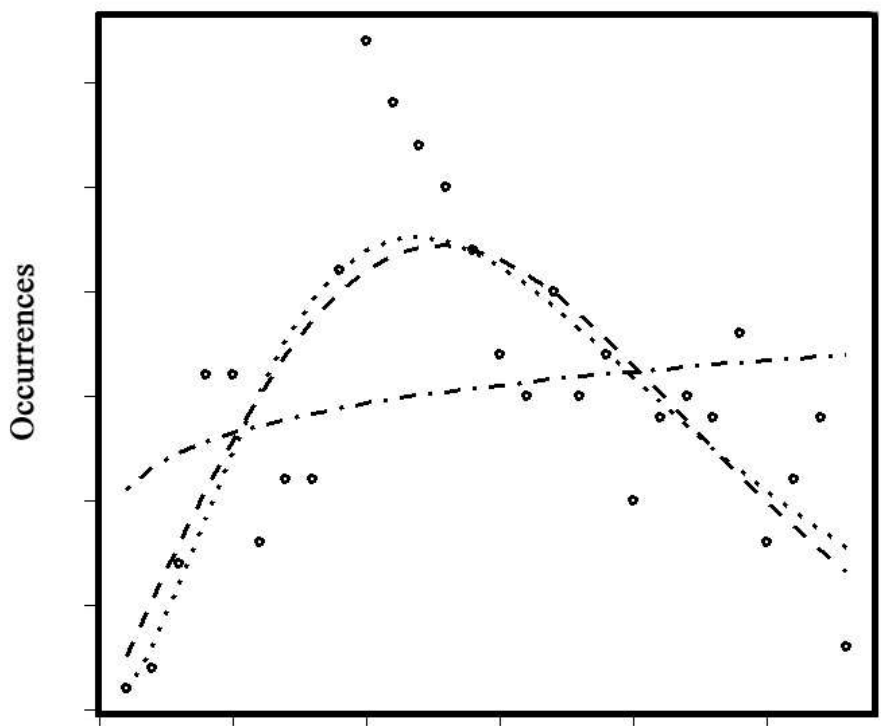

Time

Figure B2. Commercial middleware system Release i+1, which failed to fit the Exponential and Logarithmic models 


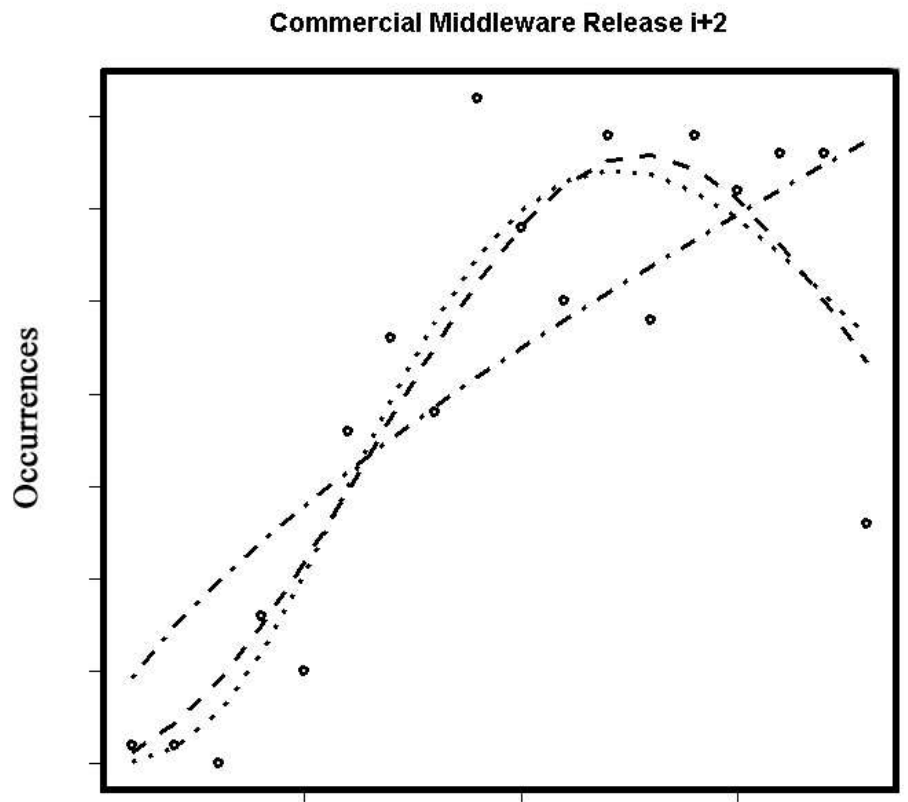

Time

Figure B3. Commercial middleware system Release i+2, which failed to fit the Exponential and Logarithmic models

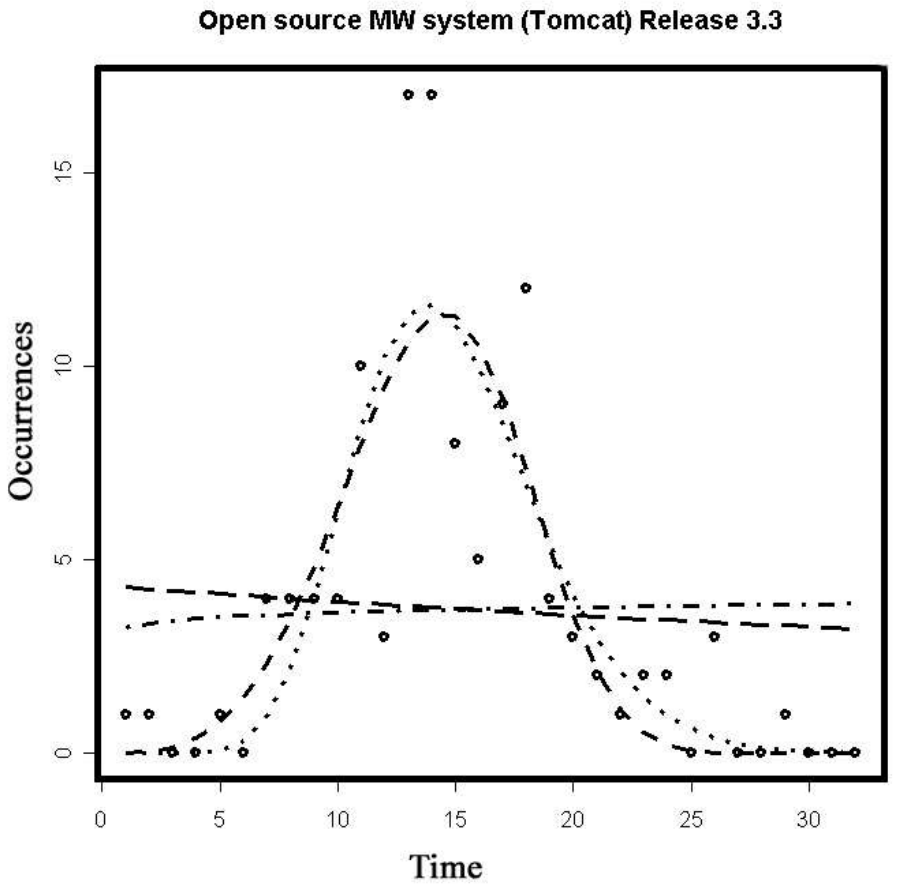

Figure B4. Open source middleware system Release 3.3, which failed to fit the Exponential model 


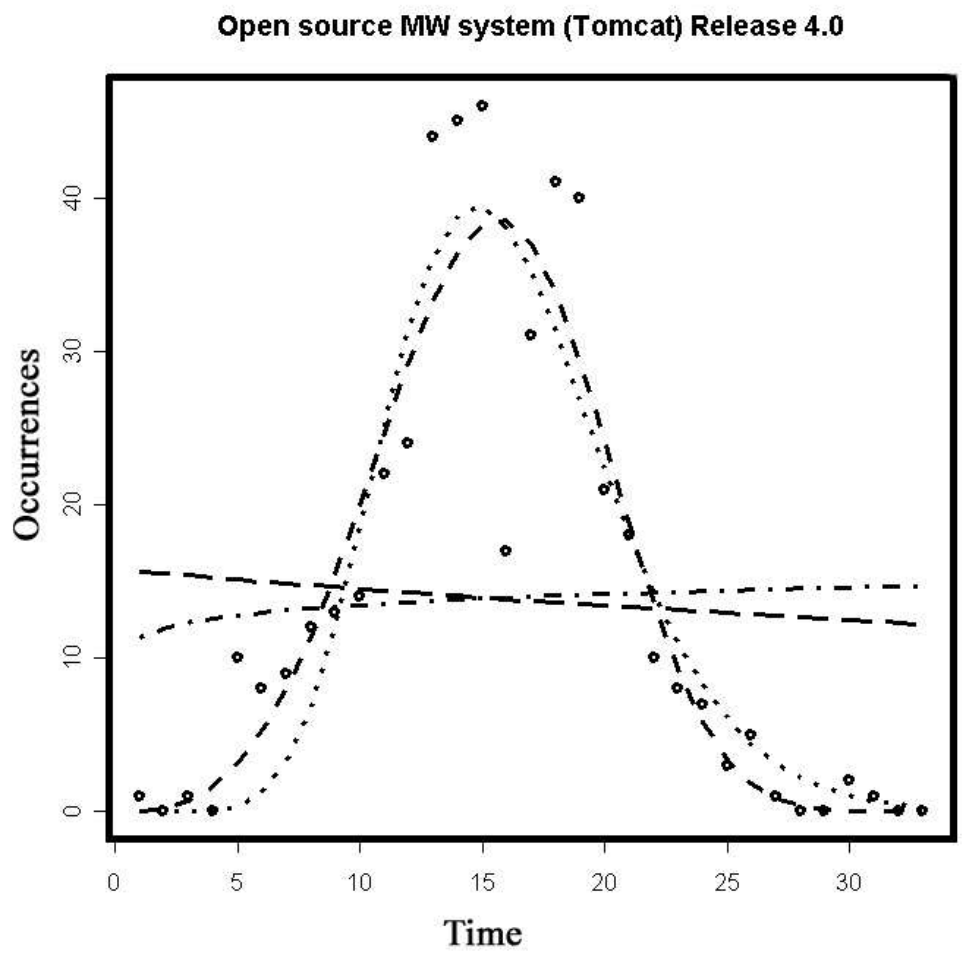

Figure B5. Open source middleware system Release 4.0, which failed to fit the Exponential model

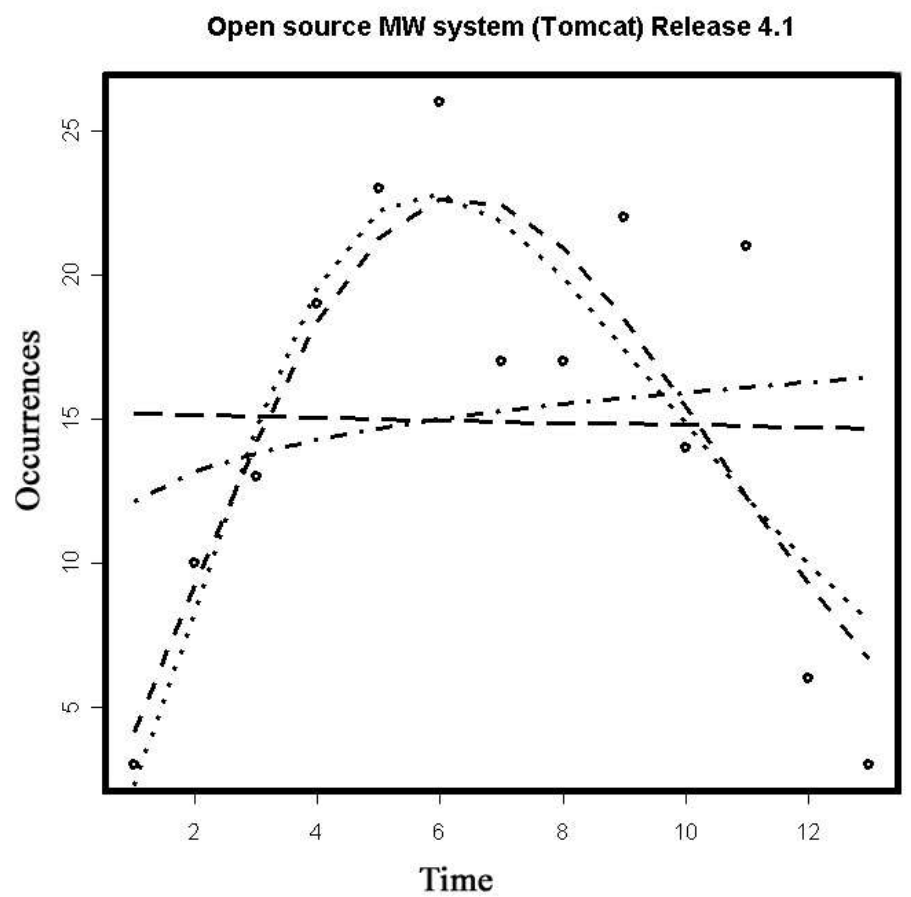

Figure B6. Open source middleware system Release 4.1, which failed to fit the Exponential model B1. The exponential model

The exponential model has the model form:

$$
\lambda(t)=N \alpha e^{-\alpha t}, \alpha>0, t>0, N>0
$$


The model is strictly decreasing with respect to t. The important variable is $\alpha$, which dictates the rate of decrease. Plot of exponential models with varying $\alpha$ is shown in figure B7.

Exponential model wity varying alpha

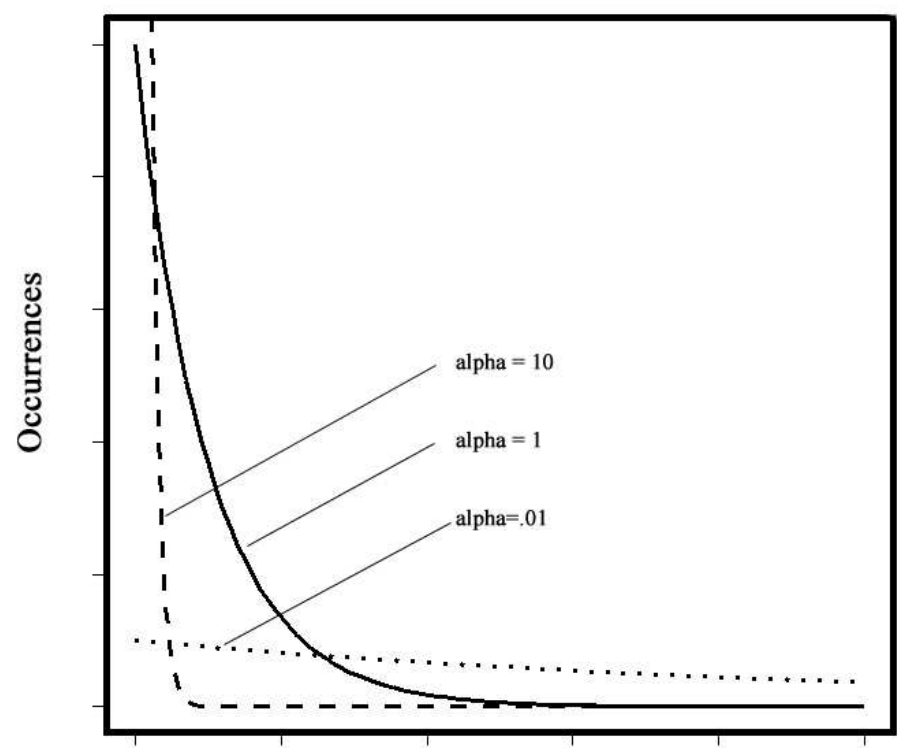

Time

Figure B7. Plots of exponential models with varying $\alpha$

There are increasing trends in releases for which the exponential model failed to fit. We use open source middleware system (Tomcat) release 4.1, which has an INF error, as an example. The plot of release 4.1 is shown in figure B8.

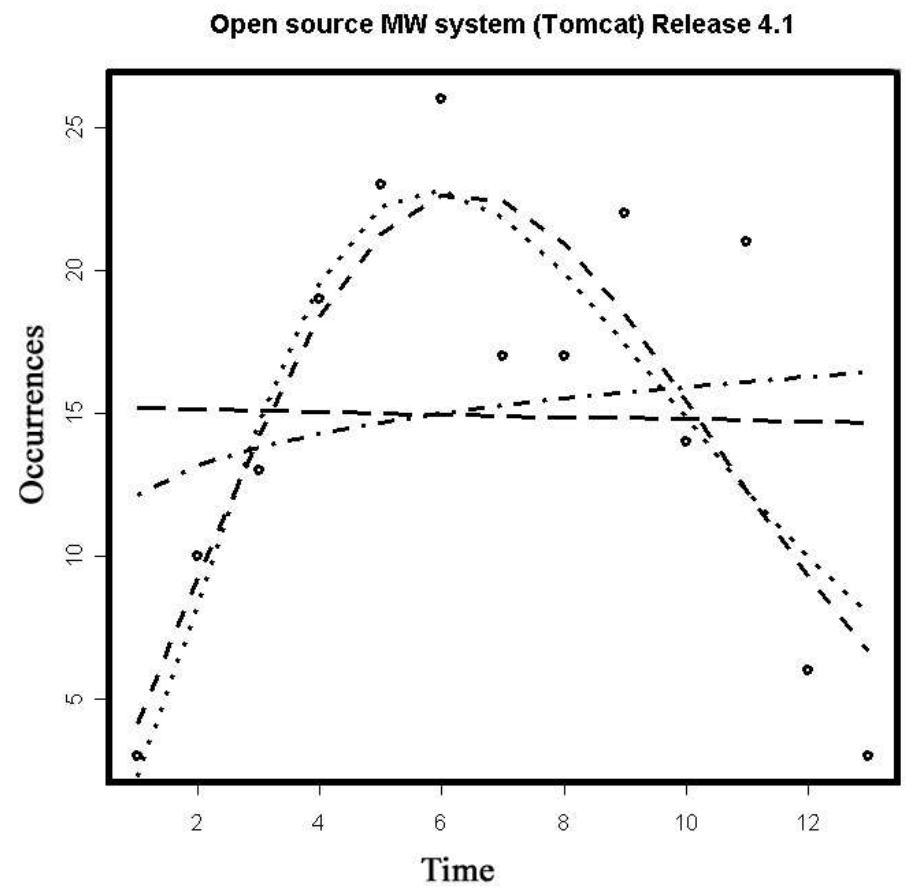

Figure B8. Plot of open source MW system Tomcat Release 4.1 
The two errors, infinity and singular gradient, indicate that the exponential model failed to be fitted to the data. The data has an increasing trend, therefore the model fitting algorithm correctly tries to minimize the residuals by reducing the rate of decrease. This translates to making $\alpha$, the variable that dictates the rate of decrease, as close to zero as possible ( $\alpha=0$ is impossible since that would mean the number of defect occurrences is zero for all time-intervals). Since $\alpha$ is outside of the exponent in the model in (1), smaller values of $\alpha$ requires correspondingly larger values of $\mathrm{N}$. The infinity error occurs when $\mathrm{N}$ is pushed toward infinity. The singular gradient error occurs when $\alpha$ is pushed towards zero, like in release 4.1 .

\section{B2. The logarithmic model}

The logarithmic model has the model form:

$$
\lambda(\mathrm{t})=\alpha(\alpha \beta \mathrm{t}+1)^{-1}, \alpha>0, \mathrm{t}>0, \beta>0
$$

The model is strictly decreasing with respect to t. The important variable is $\alpha$, which dictates the rate of decrease. Plot of logarithmic models with varying $\alpha$ is shown in figure B9.

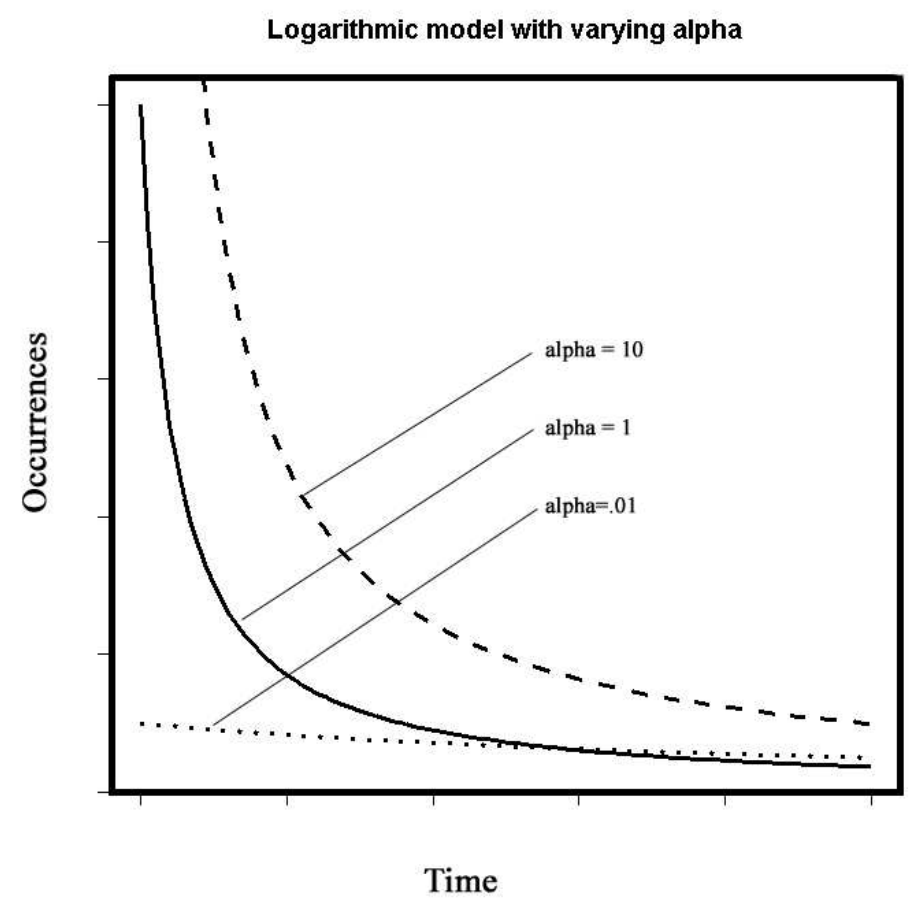

Figure B9. Plots of logarithmic models with varying $\alpha$

There are increasing trends in releases for which the logarithmic model failed to fit. We use commercial middleware release $i+2$, which has a singular gradient error, as an example. The plot is shown in figure B10. 


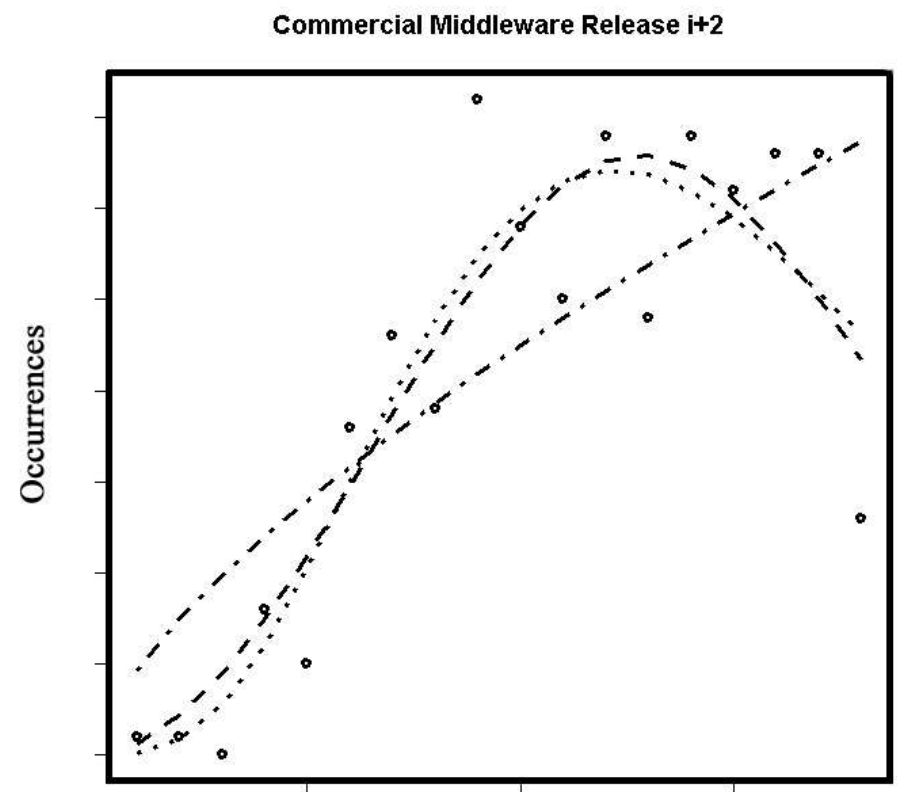

Time

Figure B10. Plot of commercial MW system Release i+2

The singular gradient error indicates that the logarithmic model failed to be fitted to the data. The data exhibits an increasing trend, therefore the model-fitting algorithm correctly tries to minimize the residuals by reducing the rate of decrease in the model. This translates into making $\alpha$, the variable that dictates the rate of decrease, as close to zero as possible ( $\alpha=0$ is impossible since that would mean the number of defect occurrences is zero for all time-intervals). The singular gradient error occurs when $\alpha$ is pushed towards zero, like in release $\mathrm{i}+2$.

\section{APPENDIX C. THE LIFETIME OF A RELEASE}

We designate the lifetime of a release as the duration of time after the release becomes generally available in which defect occurrences are being reported. Users can continue to report defect occurrences for an older release long after newer releases are available because no user can be forced to migrate away from an older release. We assume that all users eventually migrate to newer releases and no more defect occurrences will be reported an older release. The time after release when this occurs varies. Consider two releases of the open source OS in figure $\mathrm{C} 1$ and $\mathrm{C} 2$. The last defect occurrence is in the $20^{\text {th }}$ time interval for release 2.8 , but for release 2.9 the last defect occurrence is in the $15^{\text {th }}$ time interval. 


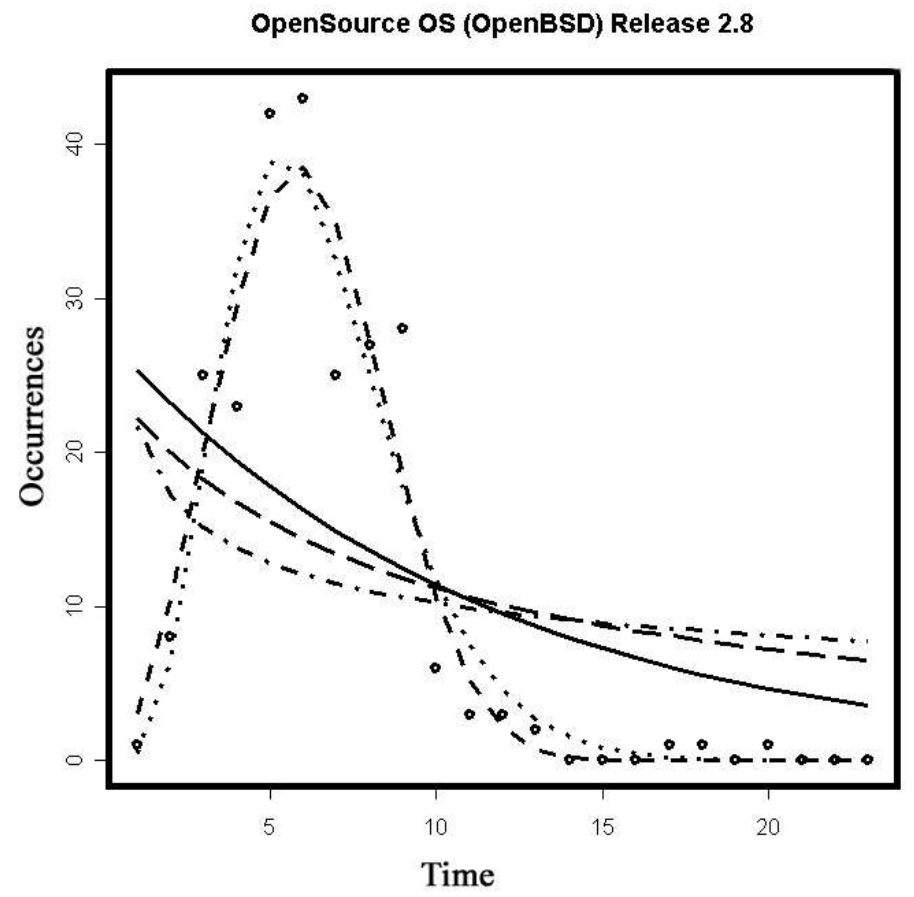

Figure C1. Plot of the open source OS Release 2.8

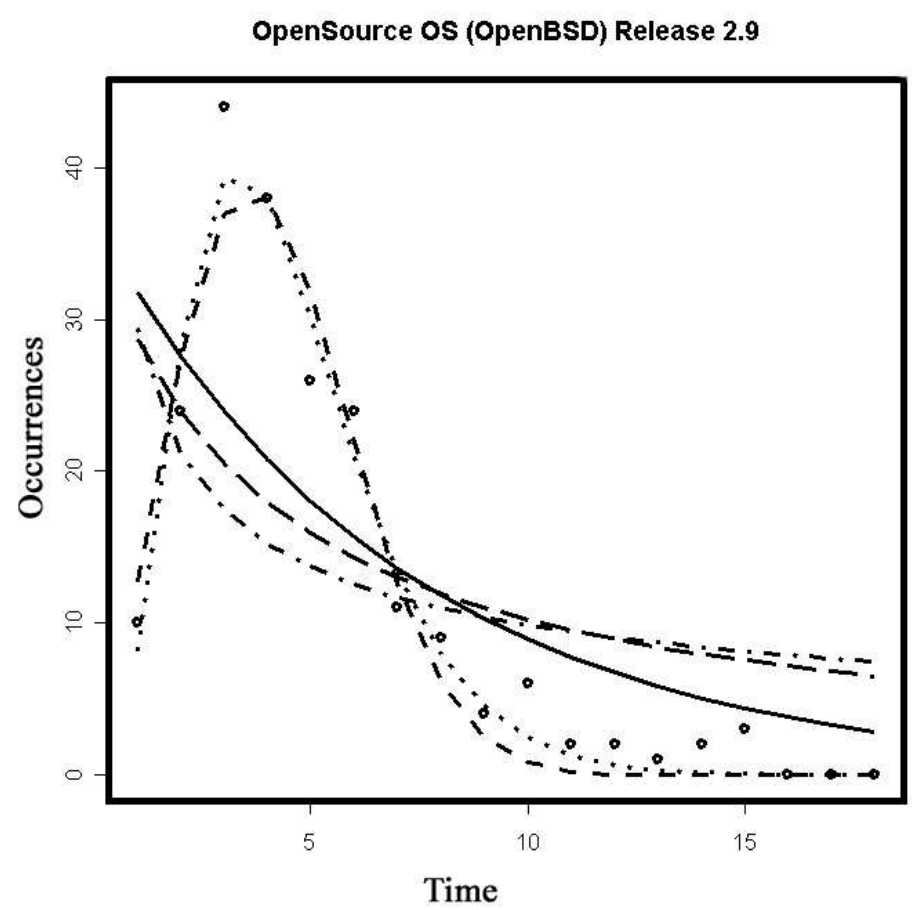

Figure C2. Plot of the open source OS Release 2.9

Operationally, we mark the end of a lifetime when we observe three successive time-intervals with zero defect occurrences. We used three successive time-intervals because releases will sometimes have more defect occurrences after several time-intervals with no defect occurrences. This is evident in the releases shown in figure $\mathrm{C} 1$ and $\mathrm{C} 2$. 
Marking the end of a release as opposed to allowing the lifetime to extend until the present time increases the validity of our results. Allowing the lifetime to continue until the present time gives an advantage to the Weibull model. Consider open source release 2.6 in figure C3. There are no more defect occurrences after the $19^{\text {th }}$ time-interval. Using the method described above, we mark the end of the lifetime at $22^{\text {nd }}$ time-interval, which produces the candidate model fits shown in figure C3.

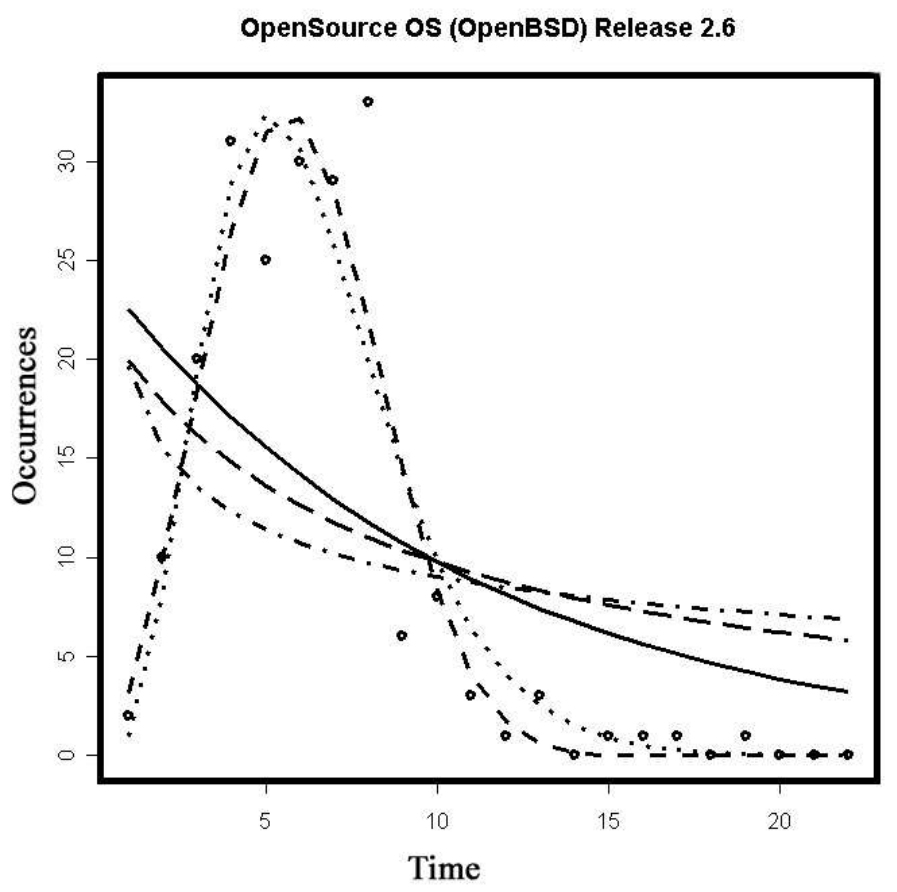

Figure C3. Plot of open source OS release 2.6

If the lifetime is extended to the present, the power, exponential, and logarithmic models would project more defect occurrences even though there are no more defect occurrences. Not marking the end of a release would offer an advantage to the Weibull model, which projects no more defect occurrences after the $22^{\text {nd }}$ time-interval. Figure $\mathrm{C} 4$ shows fitted models with the lifetime extended to the present. Table $\mathrm{C} 1$ shows the AIC scores with the extended lifetime. The results show an improvement in the Weibull model's AIC score compared with other models. 


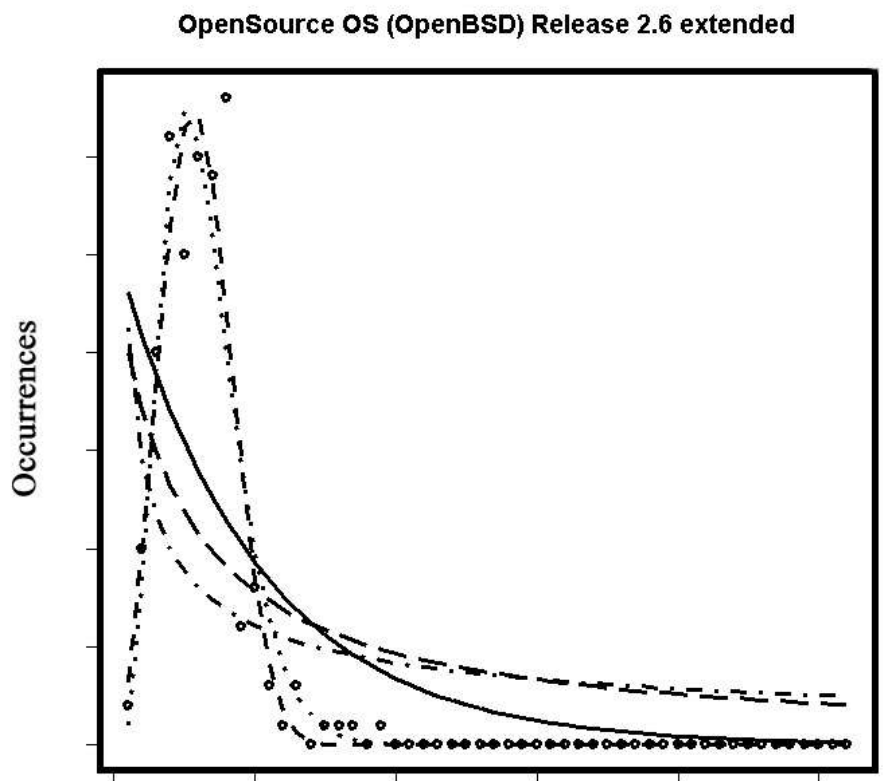

Time

Figure C4. Plot of open source OS release 2.6 with extended lifetime

Table C1. AIC scores of open source OS Release 2.6 with extended lifetime

\begin{tabular}{|c|c|c|c|c|c|}
\hline Model/Method & Exponential Model & Weibull Model & Gamma Model & Power Model & Logarithmic Model \\
\hline Extended lifetime & 345 & $\mathbf{2 4 1}$ & 252 & 367 & 358 \\
\hline Early ending lifetime & 167 & $\mathbf{1 2 4}$ & 129 & 173 & 170 \\
\hline
\end{tabular}

APPENDIX D. THE ASSUMPTION THAT THE TOTAL NUMBER OF DEFECTS IS KNOWN

We make the simplifying assumption that the total number of defect occurrences is known because it increases the validity of our results. We are interested in the defect occurrence pattern, which involves both the total number of defect occurrences and when those defect occurrences happen in the lifetime of a release. We do not want our results to be attributed to poor extrapolation of the total number of defects, since prior research have shown that two different software releases will probably have different total numbers of defect occurrences. By providing estimates of the total number of defects, we provide evidence that the characteristics discussed in section 2.2 cause the inadequacies in forecasts.

The Theil statistics in tables D1 and D2 show that results are generally even worse if we use naïve parameter extrapolation methods to extrapolate all Weibull model parameters. They now produce projections with Theil statistics greater than one in 57 out of 88 or $64.8 \%$ of the forecasting experiments. Moving averages produces forecasts with Theil statistics greater than one in 27 out of 44 or $61.36 \%$ of the forecasting experiments. Exponential smoothing method produces forecasts with Theil statistics great than one in 30 out of 44 or $68.18 \%$ of the forecasting experiments. There are now 14 out of 18 or $77.78 \%$ forecasting experiments in which the results from the using the model parameters from the most recent release result in projections that are almost no better than no change forecasts. In 32 out of 88 or $36.36 \%$ of the forecasting experiments, additional data failed to improve forecasts. These results are worse than the results in section 4.3 , which increases the validity of our results. 
Table D1. Theil forecasting statistics using moving averages method

\begin{tabular}{|c|c|c|c|c|c|c|c|}
\hline Release/System & one release & two releases & three releases & four releases & five releases & six releases & seven releases \\
\hline $\begin{array}{l}\text { Commercial } M W \\
R i+1\end{array}$ & 2.111 & & & & & & \\
\hline $\begin{array}{c}\text { Commercial } M W \\
R i+2\end{array}$ & 0.971 & 1.367 & & & & & \\
\hline $\begin{array}{c}\text { Open source MW } \\
R 4.0\end{array}$ & 1.886 & & & & & & \\
\hline $\begin{array}{c}\text { Open source } M W \\
R 4.1 \\
\end{array}$ & 2.289 & 2.091 & & & & & \\
\hline $\begin{array}{c}\text { Commercial OS } \\
\qquad i+1\end{array}$ & 1.075 & & & & & & \\
\hline $\begin{array}{c}\text { Commercial oS } \\
\quad R i+2\end{array}$ & 1.688 & 1.681 & & & & & \\
\hline $\begin{array}{c}\text { Commercial OS } \\
\quad R i+3 \\
\end{array}$ & 3.781 & 1.802 & 1.464 & & & & \\
\hline $\begin{array}{c}\text { Commercial os } \\
R i+4\end{array}$ & 0.828 & 1.133 & 0.708 & 0.571 & & & \\
\hline $\begin{array}{c}\text { Commercial oS } \\
\quad R i+5\end{array}$ & 0.989 & 1.388 & 1.433 & 1.201 & 0.895 & & \\
\hline $\begin{array}{c}\text { Commercial OS } \\
\qquad i+6\end{array}$ & 4.298 & 3.905 & 3.366 & 4.916 & 4.233 & 3.868 & \\
\hline $\begin{array}{c}\text { Commercial OS } \\
\qquad i+7\end{array}$ & 3.633 & 3.810 & 3.691 & 3.525 & 3.207 & 3.175 & 3.220 \\
\hline $\begin{array}{c}\text { Open source OS } \\
R 2.7 \\
\end{array}$ & 1.354 & & & & & & \\
\hline $\begin{array}{c}\text { Open source OS } \\
\quad R 2.8\end{array}$ & 1.101 & 0.806 & & & & & \\
\hline $\begin{array}{c}\text { Open source OS } \\
\quad R 2.9\end{array}$ & 1.453 & 0.931 & 1.039 & & & & \\
\hline Open source OS R3.0 & 0.897 & 0.558 & 0.680 & 0.696 & & & \\
\hline $\begin{array}{c}\text { Open source } O S \\
\quad R 3.1 \\
\end{array}$ & 0.797 & 0.728 & 0.760 & 0.729 & 0.738 & & \\
\hline $\begin{array}{c}\text { Open source } O S \\
\quad R 3.2\end{array}$ & 0.860 & 1.248 & 1.175 & 1.334 & 1.239 & 1.285 & \\
\hline $\begin{array}{c}\text { Open source OS } \\
R 3.3\end{array}$ & 1.469 & 1.093 & 0.967 & 0.988 & 0.843 & 0.784 & 0.702 \\
\hline
\end{tabular}


Table D2. Theil forecasting statistics using exponential smoothing method

\begin{tabular}{|c|c|c|c|c|c|c|c|}
\hline Release/System & one release & two releases & three releases & four releases & five releases & six releases & seven releases \\
\hline $\begin{array}{l}\text { Commercial } M W \\
\qquad i+1\end{array}$ & 2.111 & & & & & & \\
\hline $\begin{array}{c}\text { Commercial } M W \\
\quad R i+2\end{array}$ & 0.971 & 1.275 & & & & & \\
\hline $\begin{array}{c}\text { Open source } M W \\
R 4.0\end{array}$ & 1.886 & & & & & & \\
\hline $\begin{array}{c}\text { Open source } M W \\
R 4.1\end{array}$ & 2.289 & 2.115 & & & & & \\
\hline $\begin{array}{c}\text { Commercial OS } \\
\qquad i+1\end{array}$ & 1.075 & & & & & & \\
\hline $\begin{array}{c}\text { Commercial oS } \\
\qquad i+2\end{array}$ & 1.688 & 1.680 & & & & & \\
\hline $\begin{array}{c}\text { Commercial OS } \\
\qquad i+3\end{array}$ & 3.781 & 2.145 & 1.813 & & & & \\
\hline $\begin{array}{l}\text { Commercial OS } \\
\qquad i+4\end{array}$ & 0.828 & 0.962 & 0.730 & 0.611 & & & \\
\hline $\begin{array}{c}\text { Commercial OS } \\
\qquad i+5\end{array}$ & 0.989 & 1.318 & 1.250 & 1.185 & 1.041 & & \\
\hline $\begin{array}{c}\text { Commercial OS } \\
\qquad i+6\end{array}$ & 4.298 & 3.974 & 3.594 & 4.398 & 4.148 & 4.030 & \\
\hline $\begin{array}{c}\text { Commercial OS } \\
\qquad i+7\end{array}$ & 3.633 & 3.811 & 3.767 & 3.702 & 3.621 & 3.615 & 3.636 \\
\hline $\begin{array}{c}\text { Open source OS } \\
R 2.7\end{array}$ & 1.354 & & & & & & \\
\hline $\begin{array}{c}\text { Open source OS } \\
R 2.8 \\
\end{array}$ & 1.101 & 0.856 & & & & & \\
\hline $\begin{array}{c}\text { Open source OS } \\
R 2.9\end{array}$ & 1.453 & 1.012 & 1.067 & & & & \\
\hline Open source OS R3.0 & 0.897 & 0.574 & 0.657 & 0.657 & & & \\
\hline $\begin{array}{c}\text { Open source OS } \\
R 3.1\end{array}$ & 0.797 & 0.739 & 0.758 & 0.740 & 0.743 & & \\
\hline $\begin{array}{c}\text { Open source OS } \\
\text { R } 3.2\end{array}$ & 0.860 & 1.175 & 1.142 & 1.234 & 1.202 & 1.220 & \\
\hline $\begin{array}{c}\text { Open source OS } \\
R 3.3\end{array}$ & 1.469 & 1.163 & 1.047 & 1.041 & 0.956 & 0.931 & 0.895 \\
\hline
\end{tabular}

\section{APPENDIX E. THE DATA COLLECTION PROCESS}

The data come from IBM as summarized reports and is harvested from web-based bug reporting systems for open source software systems. IBM uses a tiered support organization described in detail by Buckley and Chillarege. We received summarized defect occurrence information from two different organizations at IBM. The data contain the release of the software systems, the time intervals, and the number of defect occurrences in each interval. The two open source software systems use web-based bug reporting systems. We obtained a copy of the mysql database containing the bug-report data for the open source middleware system. Figure E1 shows a sample bug reported for the open source middleware system (Jakarta Tomcat). We parse the bug reports into releases and time intervals using the Product, the Version, and the Opened fields circled in figure E1. 


\section{Apache Bug Database}

Bugzilla Bug $\mathbf{8 5 3}$ https:///mydomain>/securelj_security_check after successfully loging n

Query page Enter new bug

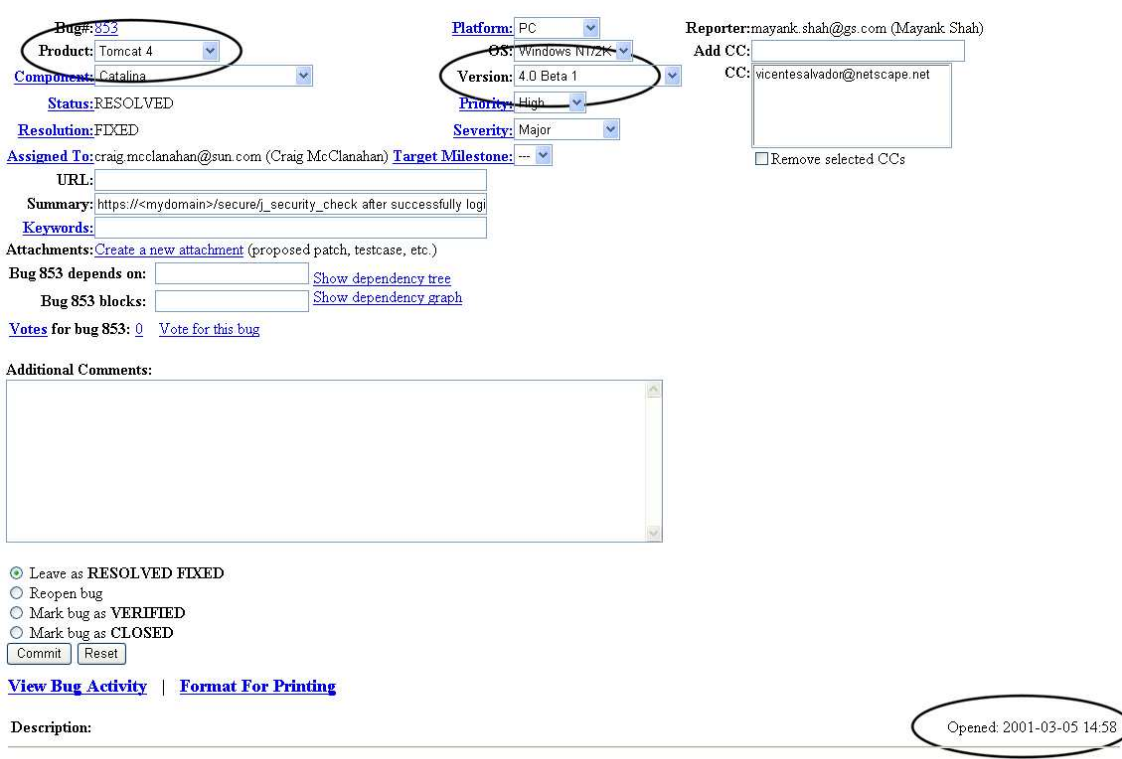

I have setup a ur 1 that is protected by tomcat. The ur 1 showen in the browser
when the loging page is rendered is the url that I was trying to reach, BUT, the url shown after successfully logging in is j_security_check. This causes all links on the pare

-----Additional Comments From Craig McClanahan 2001-03-26 12:39 -.--

What does your $\log$ in page $100 \mathrm{k}$ like? It should be submitting to

"j secur ity check" (as the servlet spec requires) so that the ultimate URL
presented will be relative to the correct directory.

For example, if your protected area is "/protected/*" and the user issues a After successful authentication, the redirected URL will be

"index heted/J_security check" so that any relative references in the

----Additional Comments From Vicente Salvador 2001-05-09 15:12 -----

But if you execute a reload on the browser, you get an error. I think that,
like tomcat 3 , a correct authentication should send a page redirect to the

original page who fires the login form page.

---Additional Comments From Vicente Salvador 2001-06-21 10:10 ---

I've analized what's the matter with this bug.

1. First what is doing now Tomcat 4

When accessing a protected URL then the scasion and request objects are stored,
then the session and request objects are cleared and initialized the request URI
to the login page URT.

The login page post to /j_security_check constant and then the original request thinks that the UR is / j security check but the content received corresponds the original page.

2. What we should do

When /j_security.check is received then redirect the browser to the original
WRL. Also we should set a flag into the session object. Wait to the browser to request the original page, then if the flag is setted
then unset it and restore the original request and session objects and let the
server continue.

This allows the browser to know than the URL it's retrieving corresnonds relly

---- Additional Comments From Craig McClanahan 2001-07-11 16:42 -...-

Fixed as recormended by vincente Salvador. Catalina now uses a redirect to

Query page Enter new bug

Actions: $\quad$ New $\mid$ Query $\mid$ Find bug \#

New account $\mid \log$ in

Figure E1. Sample bug reported for the open source middleware system 
We used web queries to gather bug report data for the open source OS. Figure E2 shows a sample bug reported for the open source OS (OpenBSD). We selected only ones that are of class, sw-bug, which signifies software bug, and parsed the bug reports into releases and time intervals using the System description in the Environment field and the Arrival-Date field circled in figure E2.

\section{OpenBSD}

Bug Query Results

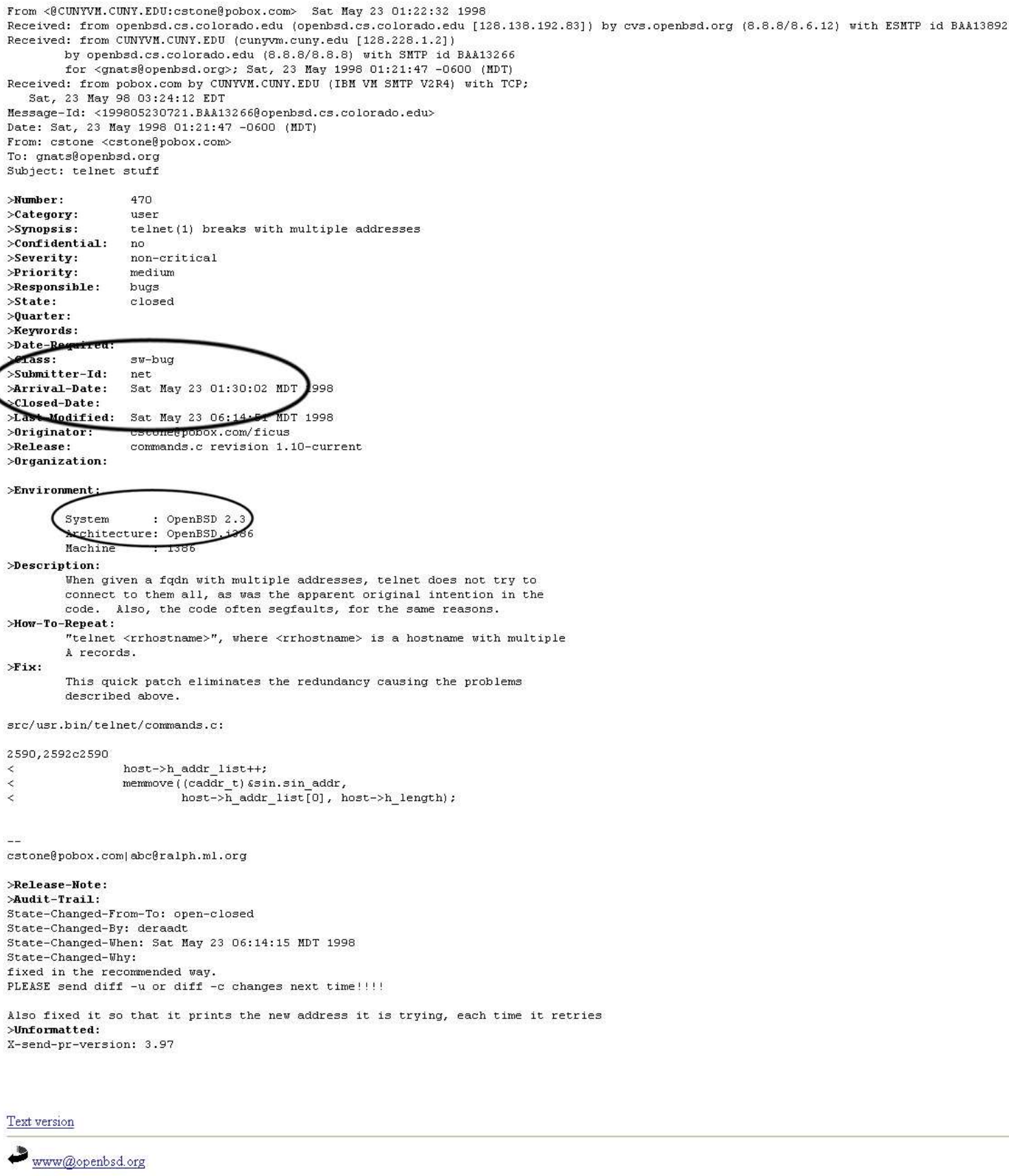

Figure E2. Sample bug reported for the open source OS 


\section{APPENDIX F. COMPARING FORECASTE ACCURACY AND NAÏVE PARAMETER EXTRAPOLATION METHOD ADEQUACY OF THE WEIBULL AND GAMMA MODELS}

We conduct another validity check on using the Weibull model as the preferred model by comparing the Theil statistic produced by the best-fit Weibull model against the Theil statistic produced by the bestfit Gamma model. We also verify that the naïve parameter extrapolation methods are inadequate by using the naïve parameter extrapolation methods outlined in section 4 to extrapolate model parameters for the Gamma model and examining the resulting Theil statistics. This investigates the possibility that extrapolating model parameters for the Weibull model is inherently difficult and that the naïve parameter estimation methods are adequate for other models.

The results show that the best-fit Weibull model produces better Theil statistics than the best-fit Gamma model in $55.56 \%$ or 10 of the 18 releases. Table F1 shows the Theil statistic of the best fit Weibull and Gamma models. In the 10 releases in which the Weibull model is better, the average advantage over the Gamma model is .103. In the 8 releases in which the Gamma model is better, the average advantage over the Weibull model is .054. These results shows that the best-fit Weibull model is the better than the best-fit Gamma model based on the Theil statistic a majority of the time. When the Gamma is better the average margin by which the Weibull model is inferior is less than the average margin when the Gamma model is inferior.

Table F2 shows the Theil statistic using the exponential smoothing method to extrapolate the model parameters for the Gamma model. In $69.813 \%$ or 74 out of 106 of the forecasting experiments, the naïve parameter extrapolation methods produce Theil statistics greater than 1 . The results are worse than the Weibull model reported in section 4.3. In $66.037 \%$ or 70 out of 106 of the forecasting experiments, the extrapolated Gamma model has worse Theil statistics than the Weibull model (shaded and bold faced values).

Table F1. Theil statistic of best fit Weibull and Gamma models

\begin{tabular}{|c|c|c|}
\hline Release & $\begin{array}{c}\text { Best fit Weibull } \\
\text { model }\end{array}$ & $\begin{array}{c}\text { Best fit Gamma } \\
\text { model }\end{array}$ \\
\hline CMW $R$ i+1 & 0.870 & 0.849 \\
\hline CMW $R$ i+2 & 0.602 & 0.593 \\
\hline OMW $R 4.0$ & 0.701 & $\mathbf{0 . 7 4 1}$ \\
\hline OMW $R 4.1$ & 0.554 & $\mathbf{0 . 5 6 8}$ \\
\hline $\operatorname{COS} R i+1$ & 0.503 & $\mathbf{0 . 6 3 5}$ \\
\hline $\operatorname{COS} R i+2$ & 0.460 & $\mathbf{0 . 5 3 5}$ \\
\hline $\operatorname{COS} R i+3$ & 0.482 & $\mathbf{0 . 4 9 7}$ \\
\hline $\operatorname{COS} R i+4$ & 0.554 & $\mathbf{0 . 5 6 4}$ \\
\hline $\operatorname{COS} R i+5$ & 0.544 & $\mathbf{0 . 5 3 9}$ \\
\hline $\operatorname{COS} R i+6$ & 0.576 & 0.576 \\
\hline $\operatorname{COS} R i+7$ & 0.547 & 0.533 \\
\hline
\end{tabular}




\begin{tabular}{|l|l|l|}
\hline OOS R2.7 & 0.319 & $\mathbf{0 . 5 4 9}$ \\
\hline OOS R2.8 & 0.478 & 0.406 \\
\hline OOS R2.9 & 0.387 & $\mathbf{0 . 4 9 9}$ \\
\hline OOS R3.0 & 0.420 & 0.303 \\
\hline OOS R3.1 & 0.676 & 0.555 \\
\hline OOS R3.2 & 0.345 & $\mathbf{0 . 6 4 3}$ \\
\hline OOS R3.3 & 0.479 & 0.351 \\
\hline
\end{tabular}

Table F2. Theil forecasting statistics using moving averages method

\begin{tabular}{|c|c|c|c|c|c|c|c|}
\hline Release/System & one release & two releases & three releases & four releases & five releases & six releases & seven releases \\
\hline $\begin{array}{c}\text { Commercial } M W \\
R i+1 \\
\end{array}$ & 2.427 & & & & & & \\
\hline $\begin{array}{c}\text { Commercial } M W \\
R i+2\end{array}$ & 0.836 & 1.227 & & & & & \\
\hline $\begin{array}{c}\text { Open source } M W \\
R 4.0\end{array}$ & 1.883 & & & & & & \\
\hline $\begin{array}{c}\text { Open source } M W \\
R 4.1\end{array}$ & 2.602 & 2.319 & & & & & \\
\hline $\begin{array}{c}\text { Commercial oS } \\
R i+1\end{array}$ & 1.145 & & & & & & \\
\hline $\begin{array}{c}\text { Commercial os } \\
\qquad i+2\end{array}$ & 1.693 & 1.718 & & & & & \\
\hline $\begin{array}{c}\text { Commercial oS } \\
\qquad i+3\end{array}$ & 3.752 & 1.769 & 1.573 & & & & \\
\hline $\begin{array}{c}\text { Commercial oS } \\
\qquad i+4\end{array}$ & 0.832 & 1.144 & 0.713 & 0.627 & & & \\
\hline $\begin{array}{c}\text { Commercial oS } \\
\qquad i+5\end{array}$ & 1.013 & 1.386 & 1.437 & 1.219 & 0.768 & & \\
\hline $\begin{array}{c}\text { Commercial oS } \\
\qquad i+6\end{array}$ & 4.281 & 3.871 & 3.311 & 4.823 & 4.159 & 3.760 & \\
\hline $\begin{array}{c}\text { Commercial oS } \\
R i+7\end{array}$ & 3.632 & 1.709 & 1.773 & 2.069 & 1.998 & 2.042 & 1.699 \\
\hline $\begin{array}{c}\text { Open source OS } \\
\quad R 2.7\end{array}$ & 1.302 & & & & & & \\
\hline $\begin{array}{c}\text { Open source OS } \\
\quad R 2.8 \\
\end{array}$ & 1.094 & 0.815 & & & & & \\
\hline $\begin{array}{c}\text { Open source OS } \\
R 2.9 \\
\end{array}$ & 1.467 & 0.956 & 1.038 & & & & \\
\hline $\begin{array}{c}\text { Open source OS } \\
\text { R3.0 }\end{array}$ & 0.912 & 0.655 & 0.751 & 0.768 & & & \\
\hline $\begin{array}{c}\text { Open source OS } \\
\quad \begin{array}{l}\text { R } 3.1 \\
\end{array}\end{array}$ & 0.744 & 0.688 & 0.724 & 0.695 & 0.705 & & \\
\hline $\begin{array}{c}\text { Open source OS } \\
R 3.2\end{array}$ & 1.212 & 1.489 & 1.377 & 1.479 & 1.364 & 1.382 & \\
\hline $\begin{array}{c}\text { Open source OS } \\
\quad \begin{array}{l}\text { R } 3.3 \\
\end{array}\end{array}$ & 1.482 & 1.331 & 1.281 & 1.251 & 1.090 & 0.995 & 0.901 \\
\hline
\end{tabular}


Table F3. Theil forecasting statistics using exponential smoothing method

\begin{tabular}{|c|c|c|c|c|c|c|c|}
\hline Release/System & one release & two releases & three releases & four releases & five releases & six releases & seven releases \\
\hline $\begin{array}{c}\text { Commercial MW } \\
R i+1\end{array}$ & 2.264 & & & & & & \\
\hline $\begin{array}{c}\text { Commercial } M W \\
R i+2 \\
\end{array}$ & 0.999 & 1.098 & & & & & \\
\hline $\begin{array}{c}\text { Open source } M W \\
R 4.0\end{array}$ & 0.942 & & & & & & \\
\hline $\begin{array}{c}\text { Open source } M W \\
R 4.1\end{array}$ & 2.065 & 2.364 & & & & & \\
\hline $\begin{array}{c}\text { Commercial OS } \\
R i+1\end{array}$ & 1.145 & & & & & & \\
\hline $\begin{array}{c}\text { Commercial oS } \\
R i+2\end{array}$ & 1.693 & 1.715 & & & & & \\
\hline $\begin{array}{c}\text { Commercial OS } \\
R i+3 \\
\end{array}$ & 3.752 & 2.111 & 1.859 & & & & \\
\hline $\begin{array}{c}\text { Commercial OS } \\
R i+4\end{array}$ & 0.832 & 0.974 & 0.736 & 0.592 & & & \\
\hline $\begin{array}{c}\text { Commercial OS } \\
\quad R i+5\end{array}$ & 1.013 & 1.317 & 1.253 & 1.196 & 0.956 & & \\
\hline $\begin{array}{c}\text { Commercial OS } \\
R i+6\end{array}$ & 4.281 & 3.941 & 3.541 & 4.316 & 5.414 & 3.947 & \\
\hline $\begin{array}{c}\text { Commercial OS } \\
\quad R i+7\end{array}$ & 3.632 & 1.857 & 1.822 & 1.963 & 2.492 & 1.920 & 1.820 \\
\hline $\begin{array}{c}\text { Open source OS } \\
R 2.7\end{array}$ & 1.302 & & & & & & \\
\hline $\begin{array}{c}\text { Open source OS } \\
R 2.8\end{array}$ & 1.094 & 0.861 & & & & & \\
\hline $\begin{array}{c}\text { Open source OS } \\
R 2.9\end{array}$ & 1.467 & 1.040 & 1.076 & & & & \\
\hline $\begin{array}{c}\text { Open source OS } \\
\text { R3.0 }\end{array}$ & 0.912 & 0.665 & 0.728 & 0.731 & & & \\
\hline $\begin{array}{c}\text { Open source OS } \\
R 3.1\end{array}$ & 0.744 & 0.696 & 0.717 & 0.702 & 0.705 & & \\
\hline $\begin{array}{c}\text { Open source OS } \\
R 3.2 \\
\end{array}$ & 1.212 & 1.442 & 1.382 & 1.433 & 1.385 & 1.387 & \\
\hline $\begin{array}{c}\text { Open source OS } \\
R 3.3\end{array}$ & 1.482 & 1.312 & 1.256 & 1.242 & 1.162 & 1.129 & 1.095 \\
\hline
\end{tabular}

